ARTICLE

https://doi.org/10.1038/s41467-018-08247-x

\title{
Single-cell analysis reveals fibroblast heterogeneity and myeloid-derived adipocyte progenitors in murine skin wounds
}

Christian F. Guerrero-Juarez ${ }^{1,2,3}$, Priya H. Dedhia ${ }^{4}$, Suoqin Jin²,5, Rolando Ruiz-Vega ${ }^{1,3}$, Dennis Ma6, Yuchen Liu'1,2,3, Kosuke Yamaga1,2,3, Olga Shestova4, Denise L. Gay ${ }^{7}$, Zaixin Yang ${ }^{8}$, Kai Kessenbrock (D) ${ }^{6}$, Qing Nie ${ }^{1,3,5}$, Warren S. Pear ${ }^{4}$, George Cotsarelis $^{8}$ \& Maksim V. Plikus (1) ${ }^{1,2,3}$

During wound healing in adult mouse skin, hair follicles and then adipocytes regenerate. Adipocytes regenerate from myofibroblasts, a specialized contractile wound fibroblast. Here we study wound fibroblast diversity using single-cell RNA-sequencing. On analysis, wound fibroblasts group into twelve clusters. Pseudotime and RNA velocity analyses reveal that some clusters likely represent consecutive differentiation states toward a contractile phenotype, while others appear to represent distinct fibroblast lineages. One subset of fibroblasts expresses hematopoietic markers, suggesting their myeloid origin. We validate this finding using single-cell western blot and single-cell RNA-sequencing on genetically labeled myofibroblasts. Using bone marrow transplantation and Cre recombinase-based lineage tracing experiments, we rule out cell fusion events and confirm that hematopoietic lineage cells give rise to a subset of myofibroblasts and rare regenerated adipocytes. In conclusion, our study reveals that wounding induces a high degree of heterogeneity among fibroblasts and recruits highly plastic myeloid cells that contribute to adipocyte regeneration.

\footnotetext{
${ }^{1}$ Department of Developmental and Cell Biology, University of California, Irvine, Irvine, CA 92697, USA. ${ }^{2}$ Sue and Bill Gross Stem Cell Research Center, University of California, Irvine, Irvine, CA 92697, USA. ${ }^{3}$ Center for Complex Biological Systems, University of California, Irvine, Irvine, CA 92697, USA.

${ }^{4}$ Department of Pathology and Laboratory Medicine, Abramson Family Cancer Research Institute, Institute for Medicine and Engineering, University of Pennsylvania, Philadelphia, PA 19104, USA. ${ }^{5}$ Department of Mathematics, University of California, Irvine, Irvine, CA 92697, USA. ${ }^{6}$ Department of Biological Chemistry, University of California, Irvine, Irvine, CA 92697, USA. ${ }^{7}$ CEA/INSERM Inserm_U967, 92265 Fontenay-aux-Roses cedex, France. ${ }^{8}$ Department of Dermatology, Kligman Laboratories, Perelman School of Medicine at the University of Pennsylvania, Philadelphia, PA 19104, USA. Correspondence and requests for materials should be addressed to G.C. (email: cotsarel@pennmedicine.upenn.edu) or to M.V.P. (email: plikus@uci.edu)
} 
S kin forms the outermost layer of the body, and principally consists of a stratified epidermis residing on top of a collagen-rich dermis. While epidermis endows skin with its barrier function, dermis provides mechanical strength and houses numerous epidermal appendages, principally hair follicles and sweat glands. Hair follicles are complex epithelial-mesenchymal mini-organs that are rich in stem cells and regenerate cyclically. When fully grown, hair follicles span the entire dermis and part of the dermal white adipose tissue (dWAT), where they engage in signaling crosstalk. As a result of this crosstalk, hair follicles induce adipocyte progenitor proliferation and adipocyte hypertrophy ${ }^{1}$. Reciprocally, dWAT modulates hair stem cell quiescence and activation ${ }^{2,3}$.

Upon significant injury, such as full-thickness excisional wounding, skin undergoes repair. While small wounds, $<1 \mathrm{~cm}^{2}$, typically repair by forming scar devoid of epidermal appendages and fat, large wounds, larger than $1 \mathrm{~cm}^{2}$, can regenerate de novo hair follicles ${ }^{4}$ and adipocytes in their center ${ }^{5}$. Large wounds in mice heal primarily by contraction, while the uncontracted portion closes by re-epithelialization and forms new connective tissue, rich in fibroblasts. In our model, wounds close in two weeks, and then new hair follicles regenerate in the central region by week three ${ }^{4,6}$, followed by new adipocytes during the fourth week ${ }^{5}$. The process of de novo hair follicle regeneration, termed wound-induced hair neogenesis (WIHN), involves reactivation of embryonic hair development programs ${ }^{4}$. Similarly, the process of de novo fat regeneration involves reactivation of an embryonic adipose lineage formation program ${ }^{5}$ (Supplementary Figure 1). It remains unclear why regeneration is limited to the wound center. Beyond laboratory mice ${ }^{4,6,7}$, WIHN is observed in rodents from the genus $A$ comys ${ }^{8}$ and in rabbits ${ }^{9}$. WIHN also likely occurs in sheep ${ }^{10}$, but not in laboratory rats ${ }^{11}$. While typically wounds in humans heal without regeneration, de novo hair follicles in facial skin wounds have been reported ${ }^{12}$.

Over the last decade, many signaling pathways necessary for WIHN have been partially elucidated. Activation of canonical WNT signaling is necessary for $\mathrm{WIHN}^{4,6,13}$, and both epidermal ${ }^{13}$ and dermal wound cells secrete and respond to WNT ligands at distinct phases ${ }^{6}$. Production of WNT ligands by dermal wound cells is initiated by FGF9, secreted by $\gamma \delta \mathrm{T}$ cells ${ }^{6}$. Activation of Hedgehog signaling downstream of WNT is necessary for dermal papilla (DP) fate specification by wound fibroblasts and for successful WIHN ${ }^{14}$. Macrophages also promote WIHN by secreting TNFa, which, in turn, activates $\mathrm{p}$-AKT/p- $\beta$-catenin signaling ${ }^{15}$. Activation of TLR3 signaling by double-stranded RNA augments IL6 production and STAT3 activation, both of which positively impact WIHN efficiency ${ }^{7}$. In contrast, prostaglandin PDG2 inhibits hair follicle neogenesis ${ }^{16}$. Furthermore, WIHN is modulated by several transcriptional regulators, including homeobox factor MSX2 ${ }^{17}$, zinc finger protein $\mathrm{CXXC5}^{18}$, and RNA-binding protein $\mathrm{MSI} 2^{19}$. De novo fat regeneration is driven by BMP ligands produced by neogenic hair follicles ${ }^{5}$. Responding to BMP signals, wound myofibroblasts activate ZFP423, a transcriptional regulator that drives adipogenic lineage commitment. Hair follicles are critical for fat neogenesis and no adipocytes regenerate in hairless wounds ${ }^{5}$.

Less is known about the lineage origin of cells during WIHN. Fate mapping experiments by Ito, Yang ${ }^{4}$ showed that progeny of the pre-existing KRT15-positive hair follicle bulge stem cells do not give rise to de novo hair follicles. Instead, progeny of nonbulge LGR6-positive ${ }^{20}$ and LGR5-positive epithelial stem cells ${ }^{15}$ can contribute toward neogenic hair follicles. Little is known about the cells that regenerate DP, the principal mesenchymal component of hair follicles. Lineage studies suggest that myofibroblasts are the source for neogenic $\mathrm{DPs}^{5,14}$. Tracing experiments on CD133-positive DP cells of preexisting hair follicles indicate that they do not mobilize upon wounding ${ }^{21}$. Other fate mapping studies suggest that multiple skin fibroblast lineages mobilize to repair small wounds ${ }^{22-24}$. Using lineage tracing with En1 (Engrailed 1)-Cre, Rinkevich, Walmsley ${ }^{23}$ identified En1-positive and En1-negative dermal fibroblasts. After wounding, En1-positive fibroblasts become major contributors toward wound repair, and genetically depleting them leads to scar reduction. The contribution of En1-positive fibroblasts toward wound repair was confirmed by Shook, Wasko ${ }^{24}$. Driskell, Lichtenberger ${ }^{22}$ showed that dermal fibroblasts contribute to wound repair in two waves. Progeny of lower dermal fibroblasts contribute early, while progeny of upper fibroblasts migrate into the wound later. Contribution from distinct dermal fibroblasts toward regenerating DPs in the WIHN model requires further investigation, yet the lack of clear lineage master-regulators complicates functional validation of DP fate mapping.

Unlike DPs, fat tissue with its well-established master-regulators, including ZFP423, CEBPs, and PPAR $\gamma$, provides a tractable model system for studying de novo regeneration. Recently, we showed that de novo adipocytes regenerate from Sm22 (aka Tagln) and Sma (aka Acta2) positive myofibroblasts ${ }^{5}$. Myofibroblast-specific ablation of Ppary or BMP receptor 1a (Bmpr1a) largely prevented adipocyte regeneration in otherwise hair-bearing wounds. However, the degree of wound myofibroblast heterogeneity and their competency for adipogenic reprogramming remains unclear. The advent of single-cell RNAsequencing (scRNA-seq) enables profiling of cellular heterogeneity in tissues with poorly characterized cell types. In this study, using a scRNA-seq approach, we identify and characterize multiple distinct fibroblast populations in regenerating mouse wounds. We show that major populations co-exist in wounds across the time course of regeneration. Furthermore, we identify bone marrow-derived adipocytes and a rare subset of wound fibroblasts with myeloid characteristics that undergo fat regeneration.

\section{Results}

Single-cell analysis reveals heterogeneity in large wounds. We performed scRNA-seq on unsorted cells from wound dermis 12 days post-wounding (PW) (Fig. 1a). This time point coincides with completion of wound re-epithelialization and strong SMA expression $^{5}$. Approximately 21,819 sequenced cells met quality control metrics (Supplementary Figure 2) and were analyzed. Unsupervised clustering using the Seurat package ${ }^{25}$ identified 13 cell clusters (Fig. 1b, left). Using the differentially expressed gene signatures, we attributed clusters to their putative identities (Fig. 1b, right) and hierarchical similarities (Fig. 1c; Supplementary Figure 3a). Figure 1d provides a summary diagram of identified cell types. Figure 1e-g show selected differentially expressed genes in the form of a heatmap (Fig. 1e), bar charts (Fig. 1f), and feature plots (Fig. 1g). Several clusters contained immune cells. The most abundant of them, representing $\sim 16 \%$ of all cells, was cluster C3. It was enriched for myeloid markers, including C1qb, Cd14, Cd68, Lyz2, Mafb, and Pf4 (Supplementary Figure 3b; Supplementary Data 1). Cluster C7 cells were classified as T lymphocytes $(\sim 4 \%)$ and they expressed $C d 3 d, C d 81, I c o s$, Maf, Nkg7, and Thy1 (Supplementary Figure 4). Cluster C8 cells were identified as B lymphocytes $(\sim 3 \%)$ and $\mathrm{C} 12$ as dendritic cells $(\sim 1 \%)$. Two other distinct cell clusters were C5 ( 9\%) and C13 ( 1\%). Cluster C5 cells were enriched for endothelial markers Cav1, Cd34, Cd93, Ly6e, Ly6c1, and Pecam1, while cluster C13 cells-for lymphatic endothelial markers Ccl21a, Lyve1, Pdpn, and Prox1. Cluster C10 cells ( 1\%) were classified as Schwann cells based on expression of Cadm4, Dbi, Gpm6b, Itih5, and Plp1. The remaining five clusters-C1, C2, C4, C6 
a
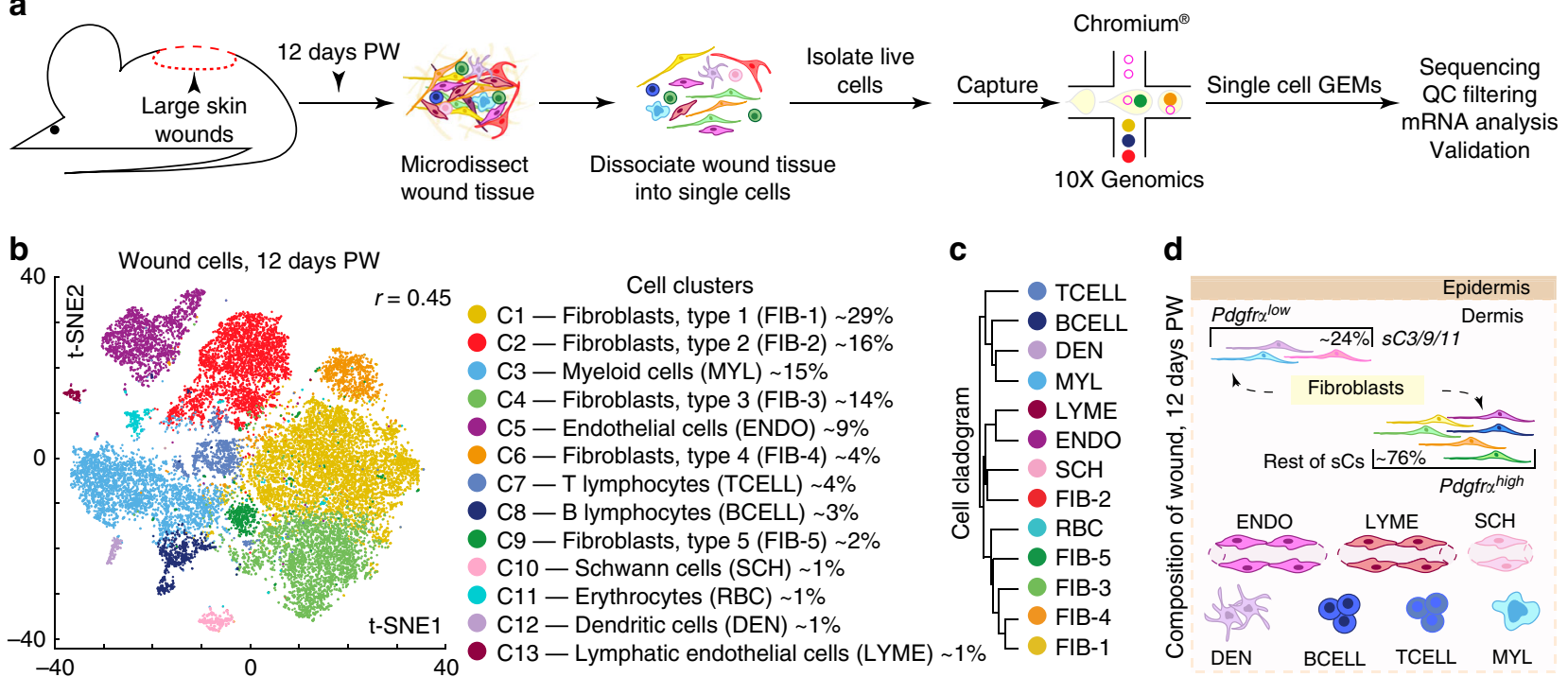

C
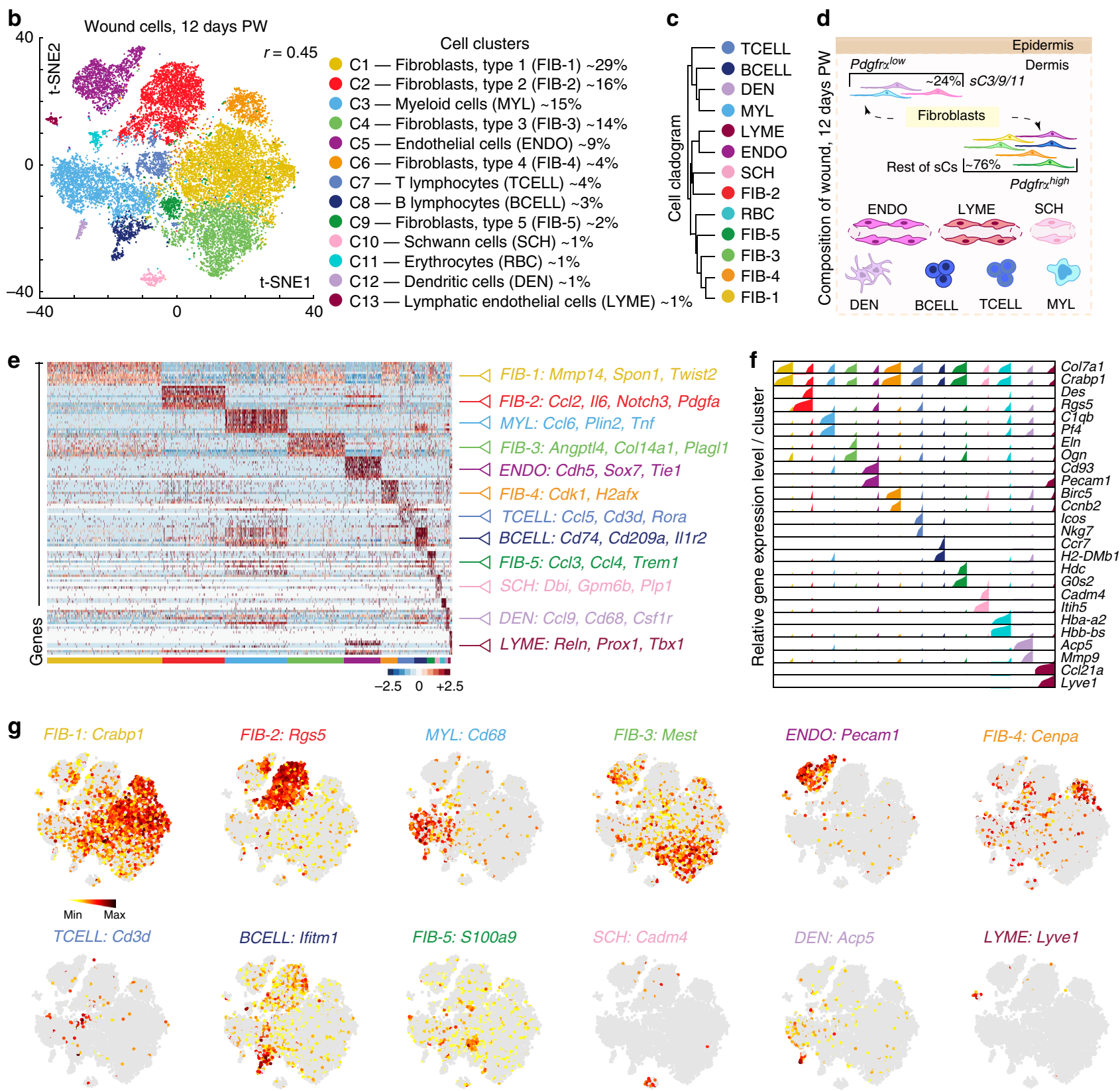

Fig. 1 scRNA-seq analysis reveals cellular heterogeneity in day 12 wounds. a Schematic of cell isolation, cell processing, capture by droplet-based device, sequencing, and downstream analysis. b t-SNE plot revealed cellular heterogeneity with 13 distinct clusters of cells identified and color-coded. General identity of each cell cluster is defined on the right. Parameter $r$ refers to Seurat's FindClusters function and determined clustering resolution.

c Unsupervised hierarchical clustering of average gene signatures showing relatedness of cell clusters (correlation distance metric, average linkage). d Wound schematic showing cellular repertoire. Different cell types, as identified on scRNA-seq, are color-coded to match colors on $\mathbf{b}$. e Heatmap of differentially expressed genes. For each cluster the top 10 genes and their relative expression levels in all sequenced wound cells are shown. Selected genes for each cluster are color-coded and shown on the right. $\mathbf{f}$ Relative expression of selected cluster-specific genes shown as high-density bar charts. Bar height corresponds to gene's relative expression level in each cell and ordering is performed from low to high expressing cells. Two genes are shown per cluster and clusters are color-coded to match the colors in $\mathbf{b} . \mathbf{g}$ Feature plots of expression distribution for selected cluster-specific genes. Expression levels for each cell are color-coded and overlaid onto t-SNE plot. Cells with the highest expression level are colored black. PW: post-wounding, GEM: gel bead-in-emulsion, QC: quality control 
and C9, representing $\sim 65 \%$ of all analyzed wound cells, were collectively characterized as fibroblasts. They were highly enriched for collagens, including Col1a1, Col3a1, Col5a1, Col12a1, and extracellular matrix protein genes, including Dcn (Decorin) and Fbln2 (Fibulin 2) (Supplementary Figure 3b; Supplementary Data 1). Many of these cells expressed high levels of contractile myofibroblast factors: Cald1, Myl9, Tagln, and Acta2 (Supplementary Figure 3b).

Wound fibroblasts subcluster into distinct cell populations. Next, we performed unsupervised clustering on all wound fibroblasts and observed further heterogeneity with 12 subclusters, $\mathrm{sC} 1$ through $\mathrm{sC12}$ (Fig. 2a, b; Supplementary Figure 5), each containing unique marker gene profiles (Fig. 2c; Supplementary Figure 6; Supplementary Data 2). Considering that transcription factors (TFs) commonly regulate cellular characteristics, we examined their expression patterns. All fibroblasts shared the following 20 TFs: Cebpb, Egr1, Fosb, Fosl2, Hif1a, Klf2, Klf4, Klf6, Klf9, Nfat5, Nfatc1, Nfkb1, Nr4a1, Nr4a2, Pbx1, Prrx1, Runx1, Stat3, Tcf4, and Zeb2 (Supplementary Figure 7a). These can be defined as a common wound fibroblast TF signature. Among these factors were Runx1 ${ }^{26}$, Tcf4 ${ }^{27}$, and Zeb2 ${ }^{28}$ (Supplementary Figure 8), previously implicated in myofibroblast differentiation. The Ebf1 high/Id $3^{\text {high }} / \mathrm{Zeb}^{\text {high }} /$ En $1^{\text {low }} / \mathrm{N} f x^{\text {low }} /$ Prrx $2^{\text {low }} /$ Sox9off signature marked fibroblasts from sC3/9/11 subclusters (Supplementary Figure $7 \mathrm{~b}, \mathrm{c}$ ), which are hierarchically distinct (Fig. 2b; Supplementary Figure 5). Among the remaining nine subclusters, fibroblasts in six subclusters, $\mathrm{sC1}$ and $\mathrm{sC} 4-\mathrm{sC} 8$, had low expression of $I d 2$ and Id3. Among these six En $1^{\text {high/ }}$ Id $2^{\text {low }} /$ Id $3^{\text {low }}$ subclusters, sC4 was Sox11 high, sC5-Twist $2^{\text {high }}$, sC7-Twist $1^{\text {high }} /$ Twist $2^{\text {high }} /$ Foxp1 $1^{\text {low }}$ and sC8-Nfiahigh (Supplementary Figure $7 b, c)$. Other subclusters also had their own, albeit complex TF expression signatures.

Next, we examined signaling pathway markers, receptors and ligands. Hierarchically distinct subclusters $\mathrm{sC} 3 / 9 / 11$ had the following receptor signature: $\mathrm{Mcam} \mathrm{m}^{\text {high}} / \mathrm{Pdg} f \mathrm{rb}^{\text {high }} / \mathrm{Fgfr} \mathrm{I}^{\text {low }} /$ Tgfbr $2^{\text {low }} / \mathrm{Tg}$ fbr3 $3^{\text {low }} / \mathrm{Ncam} 1^{\text {off }} / \mathrm{Pdg}$ fralow, and ligand signature: Il $6^{\text {high }} / \mathrm{Pdgfa} \mathrm{h}^{\text {high }} / \mathrm{Igf1} 1^{\text {low }} / \mathrm{Igfbp} 3^{\text {low }} / \mathrm{Mdk} \mathrm{k}_{\text {low }} / \mathrm{Dkk} 3^{\text {off }}$. Nine remaining subclusters were primarily differentiated from subclusters sC3/9/11 based on high Pdgfra expression, while having low Il6, Pdgfa, Pdgfrb and high Igf1, Mdk, Tgfbr2, Tgfbr3 expression (Supplementary Figure 7c). Additionally, among these nine subclusters, fibroblasts in sC2 were Angptl1 high, sC5 -Ccl8high/ Cxcl5 high/Grem $1^{\text {high }} /$ Spp $1^{\text {high }}$ sC6-Il1 $b^{\text {high }}$, sC7-Ccl8high/ Igfbp3 $3^{\text {low }}$ sC10-Angptl1 high $/$ Il1 $b^{\text {high }} / P f 4^{\text {high }}$ and sC12Angptl1 high/Fst high (Supplementary Figure 7b, c; Supplementary Data 2). We also profiled cell cycle state ${ }^{29}$. Intriguingly, sC4 and sC11 subclusters were prominently enriched for G2/M markers (Supplementary Figure 9). Considering hierarchical similarities (Fig. 2b), sC4 and sC11 likely represent actively cycling subsets of $\mathrm{sC1}$ and sC3 populations, respectively.

We also spatially mapped a portion of fibroblast subclusters to day 12 wound regions using immunostaining. We chose markers based on their high, yet specific expression on scRNA-seq and their membranous or intracellular localization (Fig. 2b). Fibroblasts in clusters $\mathrm{sC} 1 / 2 / 4-8 / 10 / 12$ were marked based on PDGFRA expression. Among these, cells in subclusters sC1/4/5/ $7 / 8$ were differentiated from $\mathrm{sC} 2 / 6 / 10 / 12$ cells based on CRABP1 expression. Intriguingly, $\mathrm{PDGFRA}^{+} / \mathrm{CRABP}^{+}$cells were enriched under the epidermis, while PDGFRA ${ }^{+} / \mathrm{CRABP}{ }^{\text {neg }}$ cells primarily localized to the lower dermis (Fig. 2d, e). Among CRABP $1^{+}$cells, rare sC7 cells $(\sim 3 \%)$ were also CYP26B1 ${ }^{+}$and showed scattered presence in the dermis (Fig. 2f). Among PDGFRA $^{+} /$CRABP1 ${ }^{\text {neg }}$ cells, rare sC6 cells $(\sim 4 \%)$ were G0S2 ${ }^{+}$ and were seldom found in the lower dermis (Fig. 2g), while
MEST2 ${ }^{+} \mathrm{sC} 2 / 10$ cells, which were abundant on scRNA-seq, showed broad presence throughout the lower dermis (Fig. 2h). sC3/9/11 fibroblasts were distinguished based on RGS5 expression. RGS5$^{+}$cells were present throughout wound dermis (Fig. 2i, j), and majority of them localized outside of the blood vessels.

Taken together, our analyses suggest that, upon completion of re-epithelialization on day 12 , large wounds may contain two major fibroblast populations. One population, representing $24 \%$ of wound fibroblasts, consists of three subclusters sC3/9/C11, which express low levels of TGF $\beta$ receptors Tgfbr2, Tgfbr3, and PDGF receptor Pdgfra and high levels of Pdgfrb. They also stain with RGS5 and localize throughout wound dermis. The second and more heterogeneous population, representing $\sim 76 \%$ of wound fibroblasts, consists of nine subclusters and expresses intermediate to high levels of Tgfbr2, Tgfbr3, high levels of Pdgfra, but not Pdgfrb. Notably, PDGFRA signaling is an established driver of fibrosis in multiple tissues, including fat and skeletal muscle. Pdgfra high cells also express higher levels of receptors for the TGF $\beta$ pathway, another well-established driver of fibrosis. Pdgfralow cells are also Pdgfr $b^{\text {high }}$, and Pdgfrb-expressing perivascular cells were identified as the precursors for new adipocytes in visceral fat in obesity ${ }^{30}$. Additionally, we identified a high level of previously unappreciated heterogeneity within both fibroblast populations. For instance, Pdgfrahigh fibroblasts further subdivide into upper and lower dermal cells both anatomically and by gene expression.

Analyses reveal myofibroblast differentiation trajectories. While t-SNE analysis helped to reveal heterogeneity among wound fibroblasts, we also wondered if they share common differentiation trajectories. Indeed, upon wounding, many fibroblasts differentiate into myofibroblasts and on scRNA-seq contractile markers were detected across multiple fibroblast subclusters. In large wounds, myofibroblasts also serve as the principal progenitors for fat regeneration ${ }^{5}$. Ordering of cells in pseudotime ${ }^{31}$ arranged most of fibroblasts into a major trajectory, with two minor bifurcations (Fig. 3a, top). Fibroblasts from different subclusters distributed broadly across the pseudotime space, with $P d g$ fralow sC3/9/11 cells primarily occupying the right half of the major trajectory, with the remaining half consisting of Pdgfrahigh cells (Fig. 3a, bottom). Next, we performed RNA velocity analysis, which considers both spliced and unspliced mRNA counts to predict potential directionality and speed of cell state transitions. It represents such transitions as vectors, with long vectors marking rapid differentiation events ${ }^{32}$. RNA velocity distinguished three sets of vectors, defined as paths. Path1 and Path2 vectors appear to represent differentiation of the Crabp $1^{\text {neg }}$ fraction of Pdgfrahigh cells and a fraction of Pdgfralow cells, respectively, toward a shared state surrounding minor bifurcations, that is primarily composed of $\mathrm{Crabp1}^{+} \mathrm{Pdgfra}$ high fibroblasts. Path3 vectors appear to represent differentiation of mainly Pdgfralow fibroblasts toward a distinct state (Fig. 3b). Cells expressing collagen Col14a1 and mesoderm-specific transcript Mest preferentially distributed at the beginning of all paths (Fig. 3d; Supplementary Data 3), while the contractile genes Acta2 and Tagln increased in density and expression levels toward the ends of these paths (Fig. 3e). We posit that all three RNA velocity paths may represent putative fibroblast differentiation trajectories toward myofibroblast states. We also examined pseudotime dynamics using scEpath ${ }^{33}$, which identifies pseudotime-dependent genes and arranges them into clusters. This analysis revealed five gene clusters (Fig. 3c), including multiple signaling factors and TFs (Fig. 3f, g; Supplementary Figure 10). Overlaying RNA velocity vectors onto these clusters 
a Wound fibroblasts, 12 days PW

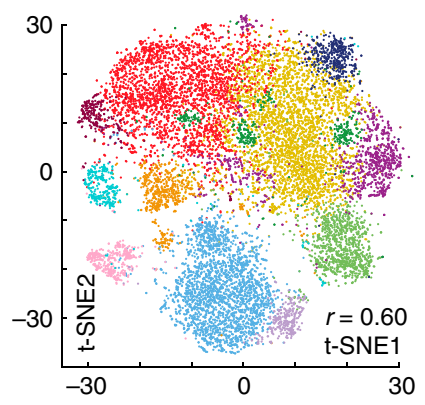

Fibroblast subclusters

sC1 - Fibroblast, type1 27\%

sC2 - Fibroblast, type2 21\%

sC3 - Fibroblast, type3 19\%

sC4 - Fibroblast, type4 7\%

sC5 - Fibroblast, type 5 7\%

sC6 - Fibroblast, type6 $\sim 4 \%$

- $\mathrm{sC7}$ - Fibroblast, type7 3\%

sC8 - Fibroblast, type8 3\%

sC9 - Fibroblast, type9 3\%

sC10 - Fibroblast, type10 2\%

sC11 - Fibroblast, type11 2\%

sC12 - Fibroblast, type12 2\% b

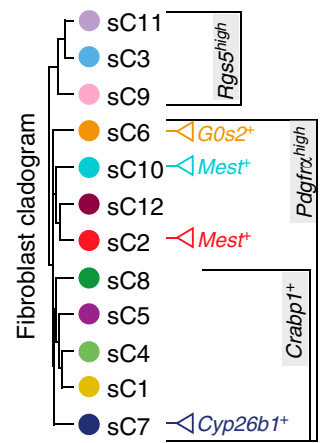

C

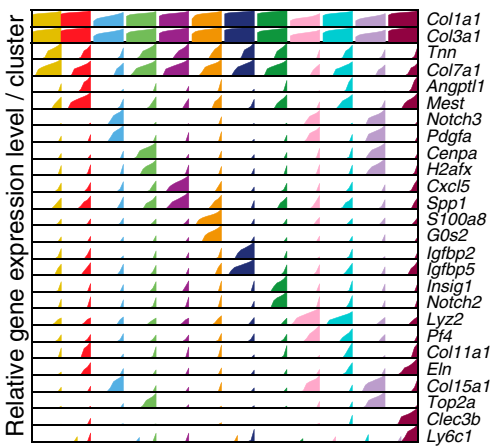

d PDGFRA CRABP1 DAPI

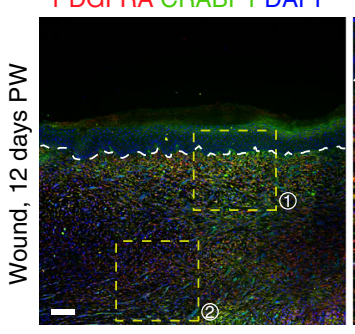

f
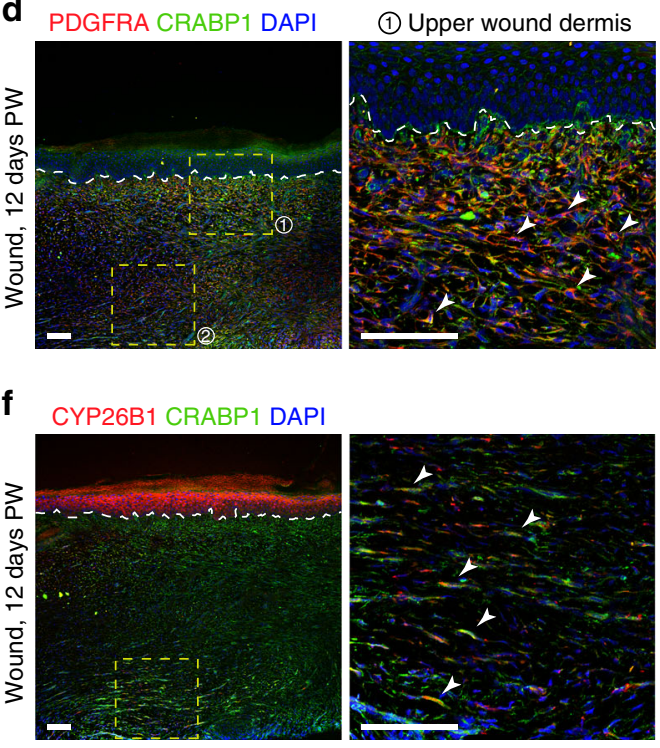

(2) Lower wound dermis

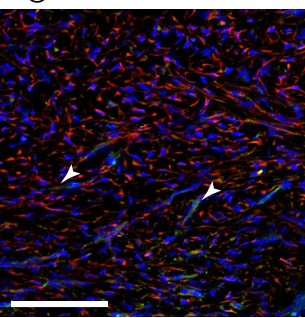

$\mathbf{g}_{\mathrm{P} p(\mathrm{r})}$

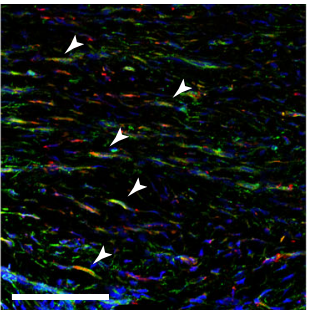

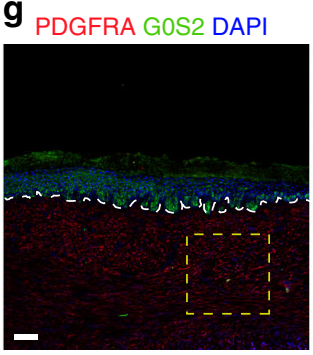

j

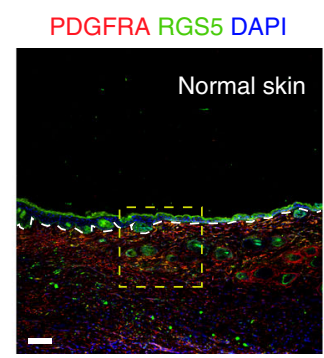

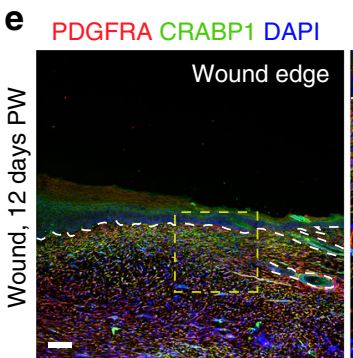

Upper wound dermis

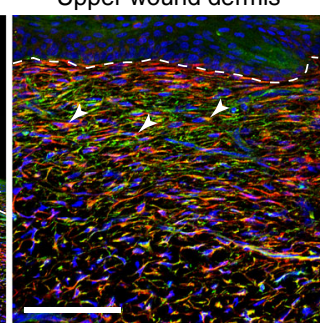

h
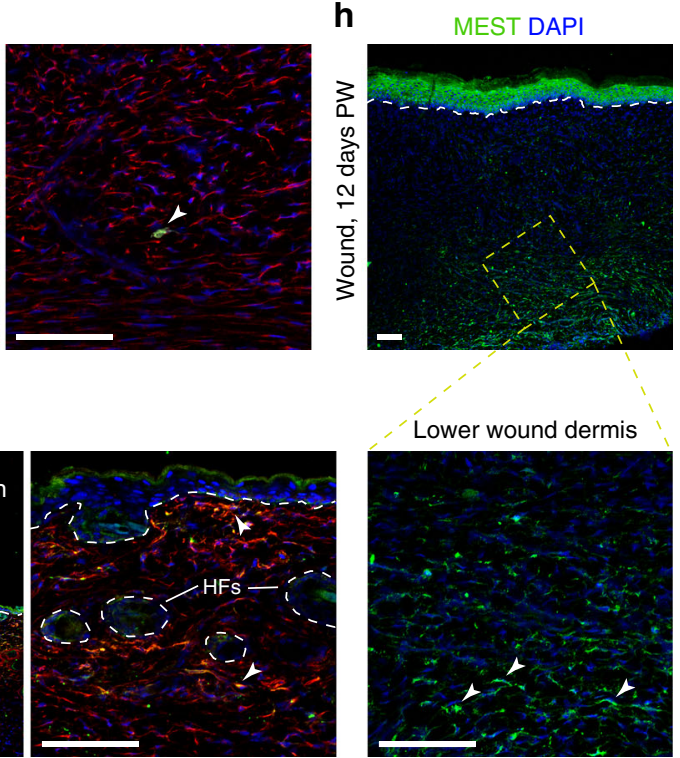

Lower wound dermis

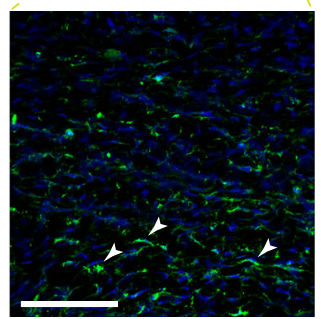

Fig. 2 Subclustering of wound fibroblasts reveals cellular heterogeneity. a Subclustering of wound fibroblasts (cells from clusters C1, C2, C4, C6, and C9 shown in Fig. 1) further identified 12 distinct subtypes. Color-coded t-SNE plot is shown and each fibroblast subcluster ( $\mathrm{s} C 1$ through $\mathrm{s} C 12$ ) is defined on the right. b Unsupervised hierarchical clustering showing relatedness of wound fibroblast subclusters. Immunostaining markers specific to individual fibroblast subclusters or groups of subclusters are listed on the right. c Relative expression of selected subcluster-specific genes shown as high-density bar charts. Bar charts are generated analogous to these in Fig. 1f. Two genes are shown per subcluster and subclusters are color-coded to match the colors in $\mathbf{a}$. $\mathbf{d}$-j $\mathbf{j}$ Staining of day 12 wounds for selected markers. d, e Co-staining for PDGFRA (red) and CRABP1 (green) identifies enriched localization of CRABP1+/PDGFRA + doublepositive fibroblasts in the upper wound dermis (arrowheads on inset 1 on $\mathbf{d}$ and inset on $\mathbf{e}$ ). Lower wound dermis contains large numbers of PDGFRA ${ }^{+}$singlepositive fibroblasts (inset 2 on d). $\mathbf{f}$ Co-staining for CRABP1 (green) and CYP26B1 (red) identifies occasional CYP26B1 ${ }^{+} / \mathrm{CRABP}^{+}$double-positive fibroblasts (arrowheads on inset). $\mathbf{g}$ Co-staining for PDGFRA (red) and GOS2 (green) identifies seldom GOS2+/PDGFRA+ double-positive fibroblasts (arrowhead on inset). $\mathbf{h}$ Numerous MEST+ cells (green) are present in the wound and primarily localize in the lower wound dermis (arrowheads on inset). $\mathbf{i}$ Co-staining for PDGFRA (red) and RGS5 (green) identifies RGS5+ single-positive fibroblasts present throughout wound dermis (arrowheads on inset). $\mathbf{j}$ Normal, unwounded skin contains both RGS5 ${ }^{+}$single-positive and RGS5+ /PDGFRA+ double-positive fibroblasts, especially in the upper dermis (arrowheads on inset). Hair follicles are marked. Immunostaining images shown in this figure are representative of the marker staining patterns observed in three or more independently stained samples. PW: post-wounding, HF: hair follicle. Size bars: $\mathbf{d}-\mathbf{j}-100 \mu \mathrm{m}$

suggests that cluster $\mathrm{pC} 1$ contains genes expressed at the beginning points of Path1 and Path2, cluster $\mathrm{pC} 2$ primarily contains Path1 differentiation program genes, cluster pC3-mainly Path2 differentiation program genes, whereas clusters pC4 and pC5 are largely composed of Path3 differentiation program genes. Taken together, pseudotime, RNA velocity, and scEpath analyses establish a basis for exploring signaling and transcriptional regulators of wound myofibroblast differentiation programs. 
a
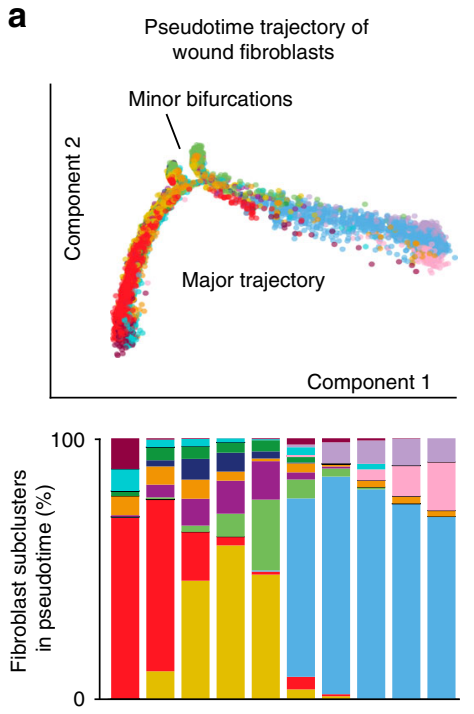

Pseudotime bins

d

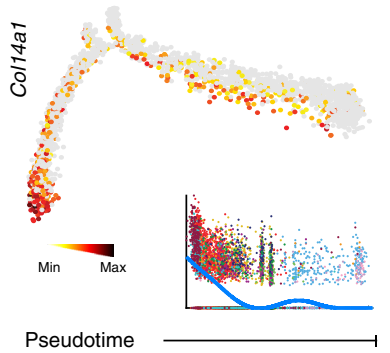

f

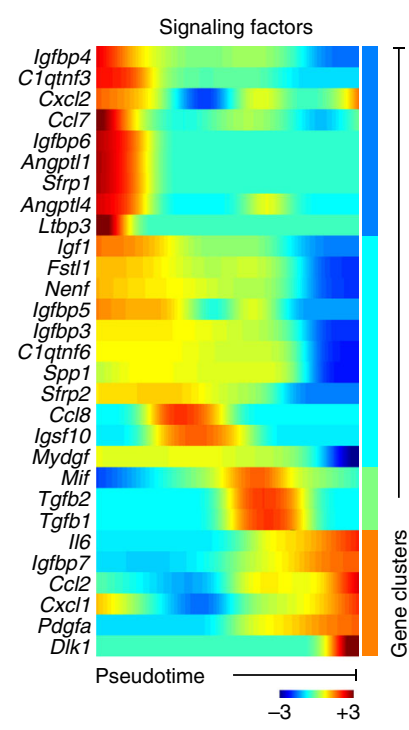

b

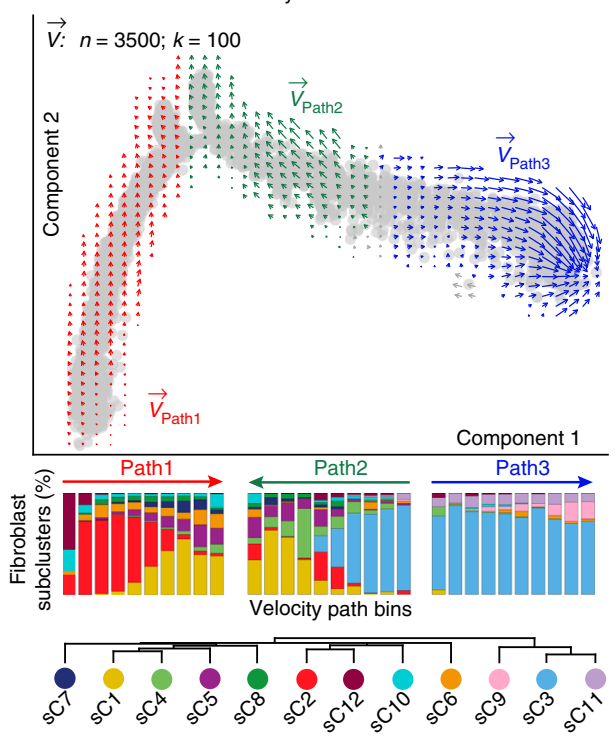

c

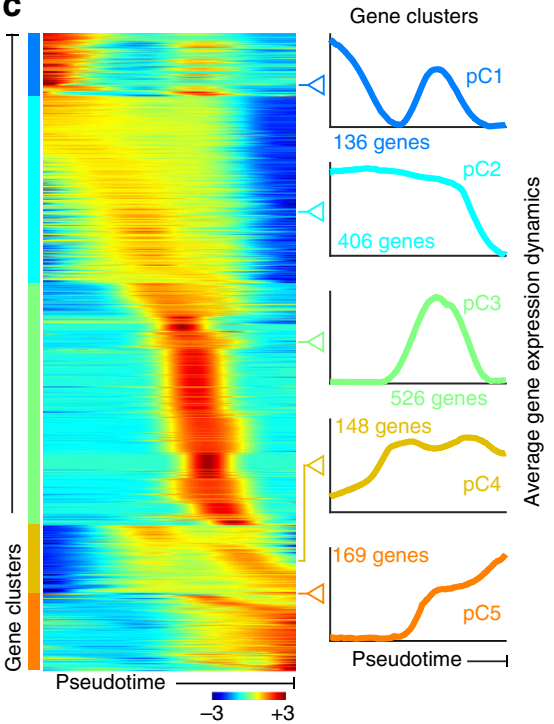

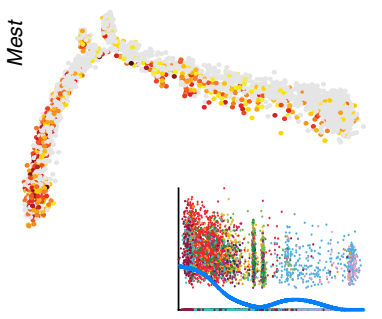

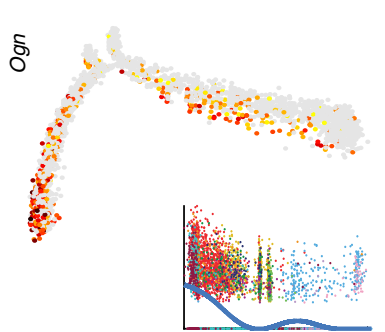

$\frac{\pi}{8}$

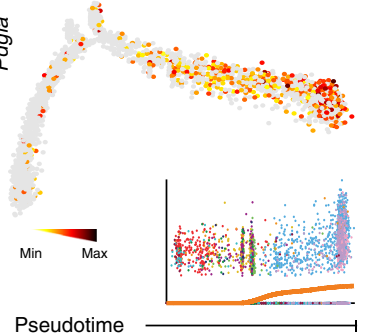

。
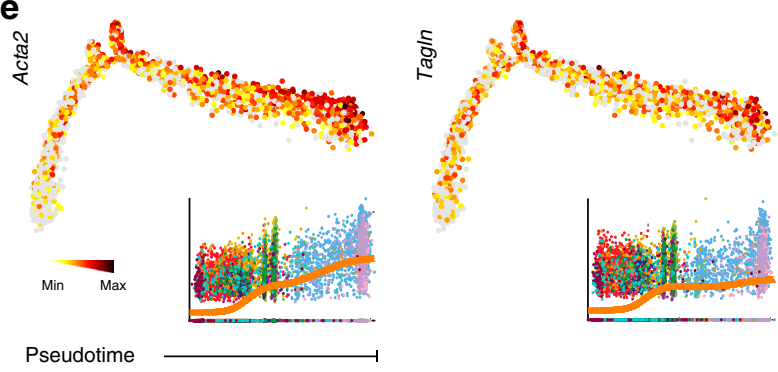

g

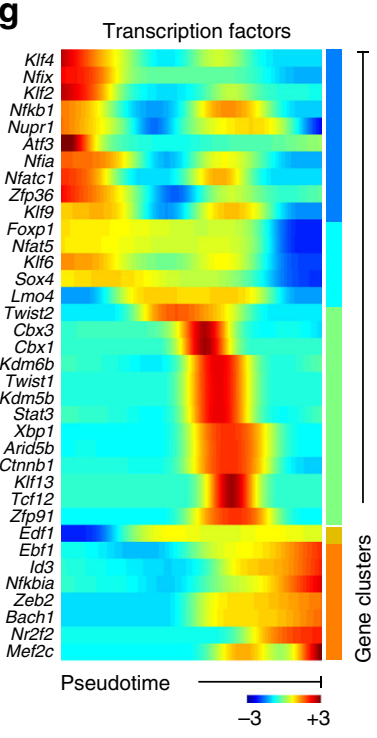

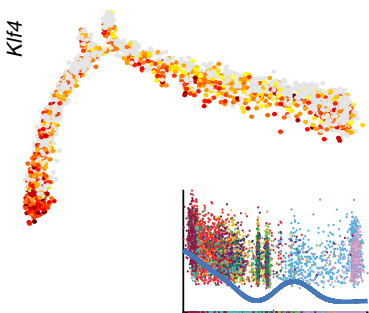

通

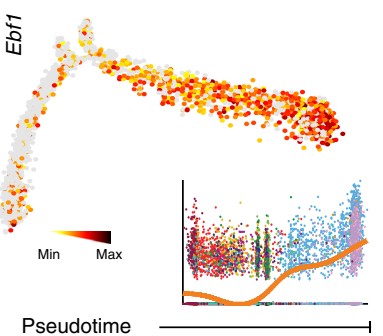

Wounds contain myofibroblasts with hematopoietic features. We noted that, on scRNA-seq, many fibroblasts across all 12 subclusters expressed hematopoietic markers. Specifically, many fibroblasts expressed the myeloid-specific marker $L y z 2$ (Lysozyme 2, aka LysM) (Fig. 4a). We examined fibroblasts that co-expressed Lyz2, collagen Col12a1 and contractile markers Acta2 and Tagln (Fig. 4b, c). This identified $\mathrm{Lyz2}^{+} / \mathrm{Acta2}^{+} /$ $\mathrm{Tagln}^{+} / \mathrm{Coll2a1}^{+}$quadruple-positive myofibroblasts, representing $\sim 11 \%$ of all wound fibroblasts. They were present in all subclusters with $\mathrm{sC} 9$ and $\mathrm{s} C 10$ being the most enriched, containing $\sim 32 \%$ and $\sim 21 \%$ of such cells, respectively (Fig. 4 c). We also used two additional markers, hematopoietic progenitorassociated $C d 34$ and myeloid-specific $C d 14$ to identify presence of $\mathrm{Cd}_{34^{+}} / \mathrm{Acta2}^{+} / \mathrm{Tagln}^{+} / \mathrm{Col12a1}^{+}$and $\mathrm{Cd} 14^{+} / \mathrm{Acta2}^{+} /$ $\mathrm{Tagln}^{+} / \mathrm{Coll2a1}^{+}$quadruple-positive myofibroblasts (Fig. 4d).

To examine if $L y z 2^{+}$myofibroblasts might derive from woundresident myeloid cells, we performed pseudotime analysis jointly on all Lyz2-expressing wound fibroblasts and myeloid cells (cells from cluster C3 on Fig. 1b). This analysis arranged all cells into a major trajectory, with several minor bifurcations (Fig. 4e). 
Fig. 3 Pseudotime analyses reveal putative fibroblast differentiation trajectories. a Pseudotime ordering on wound fibroblasts arranged them into a major trajectory, with two minor bifurcations (top panel). Stacked bar chart shows relative abundance of fibroblasts from distinct subclusters across the pseudotime, which was divided into ten equal bins (bottom panel). Pdgfralow sC3/9/11 cells primarily occupy right half of the trajectory, with the remaining part consisting of Pdgfrahigh cells. All cells on the pseudotime and the bar chart are color-coded to match the colors in Fig. 2a. b RNA velocity analysis distinguished three sets of velocity vectors across the pseudotime: Path1 (red), Path2 (green) and Path3 (blue). Stacked bar charts show relative abundance of fibroblast subclusters across individual RNA velocity paths (middle panel). Bottom panel defines fibroblast subcluster colors and matches the colors in Fig. 2a. c scEpath analysis performed on pseudotime identified five gene clusters (pC1 through pC5) of pseudotime-dependent genes. Heatmap of the expression levels for all differentially expressed genes in all analyzed wound fibroblasts is shown on the left. Average gene expression dynamics and gene counts in all five clusters are shown on the right. $\mathbf{d}$ Feature plots of expression distribution for Col14a1 and Mest across pseudotime. Both genes are part of cluster $\mathrm{pC} 1$ and mark the beginning points of RNA velocity Path1 and Path2. Expression levels for each cell are color-coded with the highest expression level colored black. e Feature plots of expression distribution for contractile markers Acta2 and Tagln across pseudotime. Cells with the highest expression level are colored black. Numbers of both $\mathrm{Acta2}^{+}$and $\mathrm{Tag}^{+}{ }^{+}$cells peak around minor bifurcations and along Path3. $\mathbf{f}, \mathbf{g}$ Expression level heatmaps for selected signaling factors $(\mathbf{f})$ and transcription factors $(\mathbf{g})$ identified as differentially expressed on $\mathbf{c}$. Each heatmap is accompanied by colorcoded pseudotime feature plots for two selected genes. Numbers of $\mathrm{Ogn}^{+}$and $\mathrm{KIf}^{+}$cells peak at the beginning points of RNA velocity Path1 and Path2, while numbers of $\mathrm{Pdgfa}^{+}$and $\mathrm{Ebfl}^{+}$cells increase along Path3. Cells with the highest expression level are colored black

Fibroblasts (orange) and myeloid cells (black) distributed differentially across the pseudotime space; however, a prominent overlap region was observed in the middle of the trajectory (Fig. 4f). Within this region, both cell types displayed hybrid expression patterns, simultaneously enriched for fibroblastspecific and myeloid-specific genes (Fig. 4g; Supplementary Figure 11a). Acta2 and Tagln were enriched in Lyz2 $2^{+}$fibroblasts, but were also expressed in $L y z 2^{+}$myeloid cells, including in the overlap region (Fig. 4h). scEpath identified five pseudotimedependent gene clusters (Fig. 4i; Supplementary Figure 11b-e; Supplementary Data 4), while RNA velocity distinguished four vector paths (Fig. 4j). Importantly, Path3 vectors, which encompass the overlap region, appear to represent putative conversion of $\mathrm{Lyz}^{+}$myeloid cells into fibroblasts (red on Fig. 4j), while Path4 vectors appear to mark differentiation of woundresident myeloid cells (green on Fig. 4j). Overlaying RNA velocity vectors onto scEpath data indicates that genes in cluster $\mathrm{rC5}$ mark myeloid differentiation program along Path4, while genes in clusters $\mathrm{rC} 4$ and $\mathrm{rC} 3$ mark early and late stages of putative myeloid-to-fibroblast conversion pathway, respectively.

Next, we performed scRNA-seq on genetically marked contractile cells, isolated from day 12 wounds of Sm22-Cre; tdTomato mice (Fig. 5a). A microfluidic-based capture platform was used (Supplementary Figure 12a). Although it captured substantially fewer cells, 116 in total, compared to the dropletbased capture approach, it yielded a higher depth of sequencing, 5830 vs. 1101 median genes/cell (Supplementary Figure 12b vs. $2 \mathrm{~b}$ ), and eliminated the possibility of cell doublets. On t-SNE analysis, cells segregated into three clusters- $\mathrm{fC} 1, \mathrm{fC} 2$, and $\mathrm{fC} 3$ (Fig. 5b), each with its distinct gene markers (Supplementary Figure 13; Supplementary Data 5). Pearson correlation showed that $\mathrm{fC} 1$ cells were the most similar to Pdgfrahigh fibroblasts from subclusters $\mathrm{sC} 1 / 4 / 5 / 8 / 10$. Cells from $\mathrm{fC} 2$ were the most similar to $P d g$ fra low fibroblasts from subclusters $\mathrm{sC} 3 / 11$ and expressed high levels of $P d g f r b$. Cluster $\mathrm{fC} 3$ had the highest correlation to sC9 subcluster (Fig. 5d), enriched for $\mathrm{Lyz}^{+} / \mathrm{Acta}^{+} / \mathrm{Tagln}$ $+/ \mathrm{Coll2}_{2} \mathrm{I}^{+}$quadruple-positive myofibroblasts. Consistently, this cluster was also the most enriched for hematopoietic markers, including pan-hematopoietic Cd45 (aka Ptprc), and myeloidspecific Lyz2 and Ccl6 (Fig. 5c; Supplementary Figure 13). To confirm Lyz2 ${ }^{+}$myofibroblasts at the protein level, we performed single-cell western blot on unsorted cells from day 12 Sm22-Cre; tdTomato wounds and stained for LYZ and tdTomato (Fig. 5e; Supplementary Figure 14). Indeed, $\sim 6 \%$ of tdTomato-expressing wound cells were $\mathrm{LYZ}^{+} /$tdTomato $^{+}$double-positive (77 out of 1293 cells) (Fig. 5f). We also co-stained day 12 wounds for LYZ and fibroblast markers PDGFRA (Fig. 5g) and SMA (Fig. 5h), and in both cases detected double-positive cells.
To determine if myeloid marker-expressing fibroblasts persist in healed wounds, we performed scRNA-seq on tdTomato ${ }^{\text {hi }}$ cells isolated from Sm22-Cre;tdTomato wounds at two additional time points, 15 days and 21 days PW (Supplementary Figure 15a). Combined analysis on all cells from 12 days, 15 days, and 21 days PW showed that they form four clusters, tCl through tC4 (Supplementary Figure 15b; Supplementary Data 6). Cells from all three time points contributed to all four clusters (Supplementary Figure 16a), although relative cluster contribution was time point-dependent (Supplementary Figures 15c and 16b). Cluster tC4 was closely related to cluster fC3 from 12 days PW analysis (Supplementary Figure 15d) and also contained Cd45 and Lyz2 expressing cells (Supplementary Figure 16c). Taken together, we identified a population of myofibroblasts with myeloid features that persist in wounds over the course of repair and regeneration and potentially form via myeloid cell reprograming.

Hematopoietic lineage cells contribute to wound dermis. Following our scRNA-seq findings, we noted that previous work indicated that myeloid cells can convert into myofibroblasts ${ }^{34}$, but the extent of this conversion is organ and injury contextspecific $^{35-41}$. Considering that in large wounds, adipocytes regenerate predominantly from myofibroblasts ${ }^{5}$, we asked to what extent myeloid cells contribute to their population. We performed wounding experiments in bone marrow transplantation (BMT) mouse models. In some BMT models, we reconstituted lethally irradiated mice with GFP-expressing cells, while in others with cells expressing lacZ under the control of lineagespecific promoters (Fig. 6a). To distinguish between hematopoietic and non-hematopoietic cells, we performed BMT using the sorted $\mathrm{CD}_{4} 5^{+}$bone marrow (BM) fraction. In $\mathrm{CD} 45^{+} \mathrm{BMT}$ models, we achieved high levels of peripheral blood chimerism, compatible to BMT mice reconstituted with whole BM (Supplementary Figure 17). We also generated BMT mice using multipotent hematopoietic stem cells (HSCs) purified based on the SLAM marker signature: Lineage ${ }^{\text {neg, }}$ SCA $1^{+}, \mathrm{c}-\mathrm{KIT}^{+}, \mathrm{CD} 150^{+}$, CD48 neg (Supplementary Figure 17e). We transplanted between 2300 and 4400 HSCs per recipient mouse and in all cases achieved successful reconstitution of the hematopoietic lineage, confirmed by high levels of peripheral blood chimerism (Supplementary Figure 17f, g) and bone marrow fluorescence (Supplementary Figure 18a). Following wounding, healed tissue in $\mathrm{GFP}^{+}$HSCs BMT mice showed consistently high contribution from hematopoietic lineage on 28 days PW, with many $\mathrm{GFP}^{+}$ cells surrounding neogenic hair follicles ( $n=18$ animals) (Fig. 7a; Supplementary Figure 19). Flow cytometry on wound tissue confirmed that long-term contribution from the hematopoietic 
a

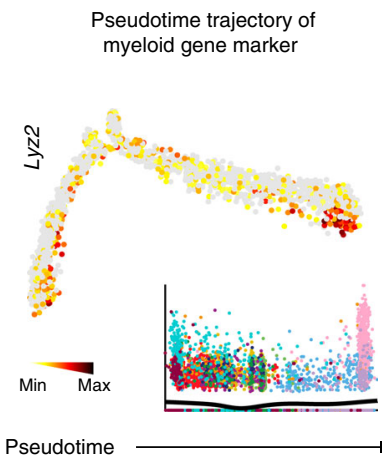

b

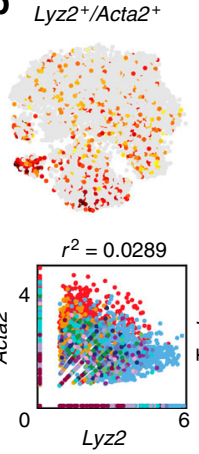

$\mathrm{Lyz2}^{+} / \mathrm{Tag} / \mathrm{n}^{+}$

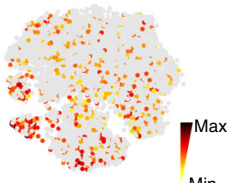

Min

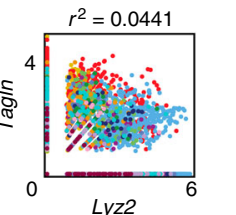

C
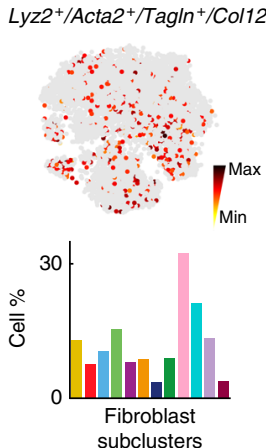

d Cd34
Tagl $^{+} /$Acta2 $^{+}$
Col12a $1^{+}$
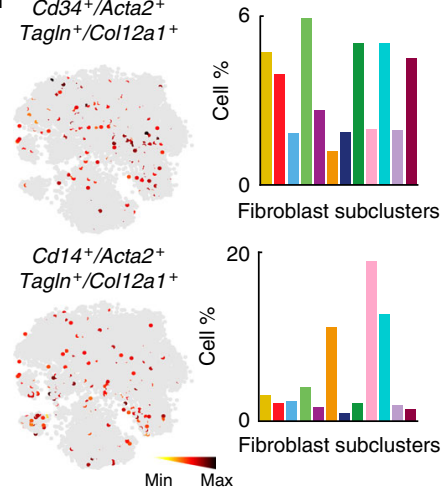

e

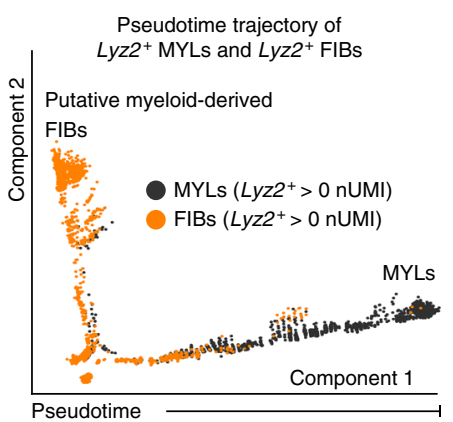

h

h Pseudotime distribution of contractile
genes in Lyz2-expressing cells

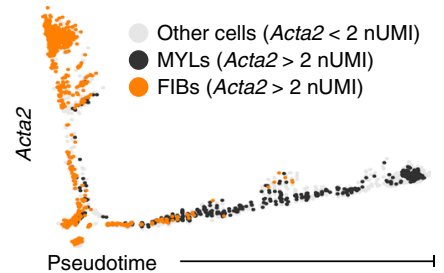

f

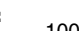

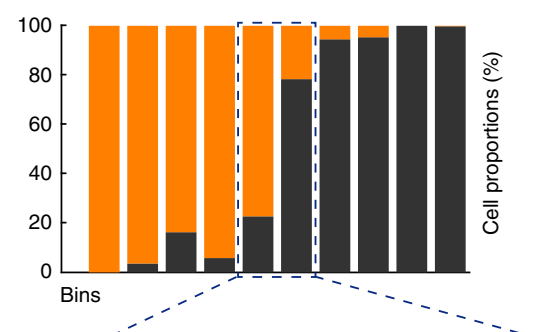
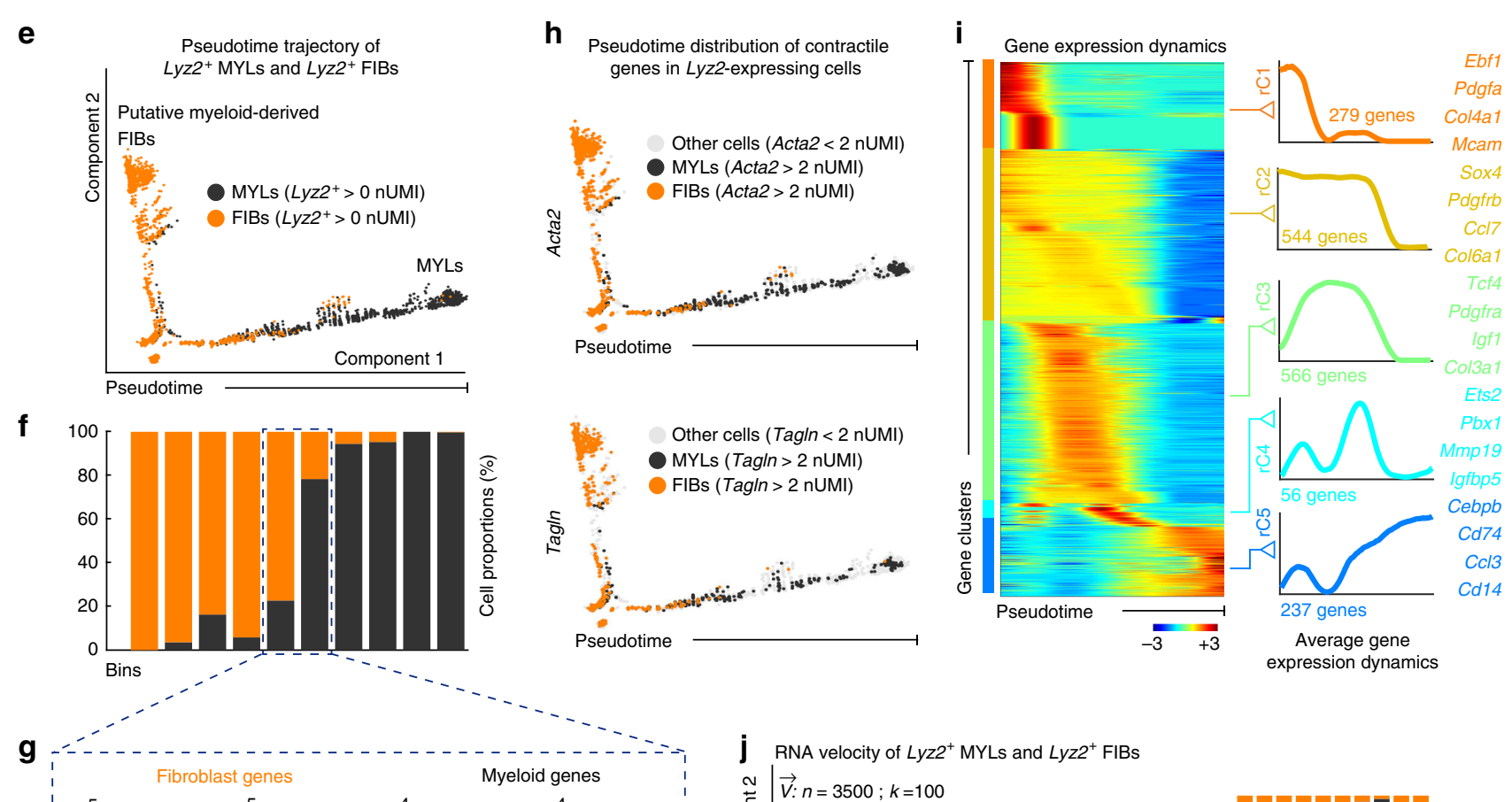

j RNA velocity of $L y z 2^{+} M Y L s$ and $L y z 2^{+}$FIBs
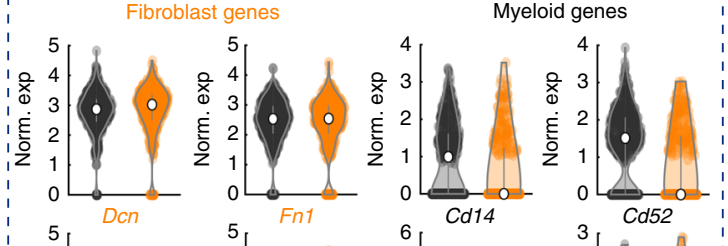

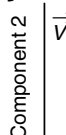
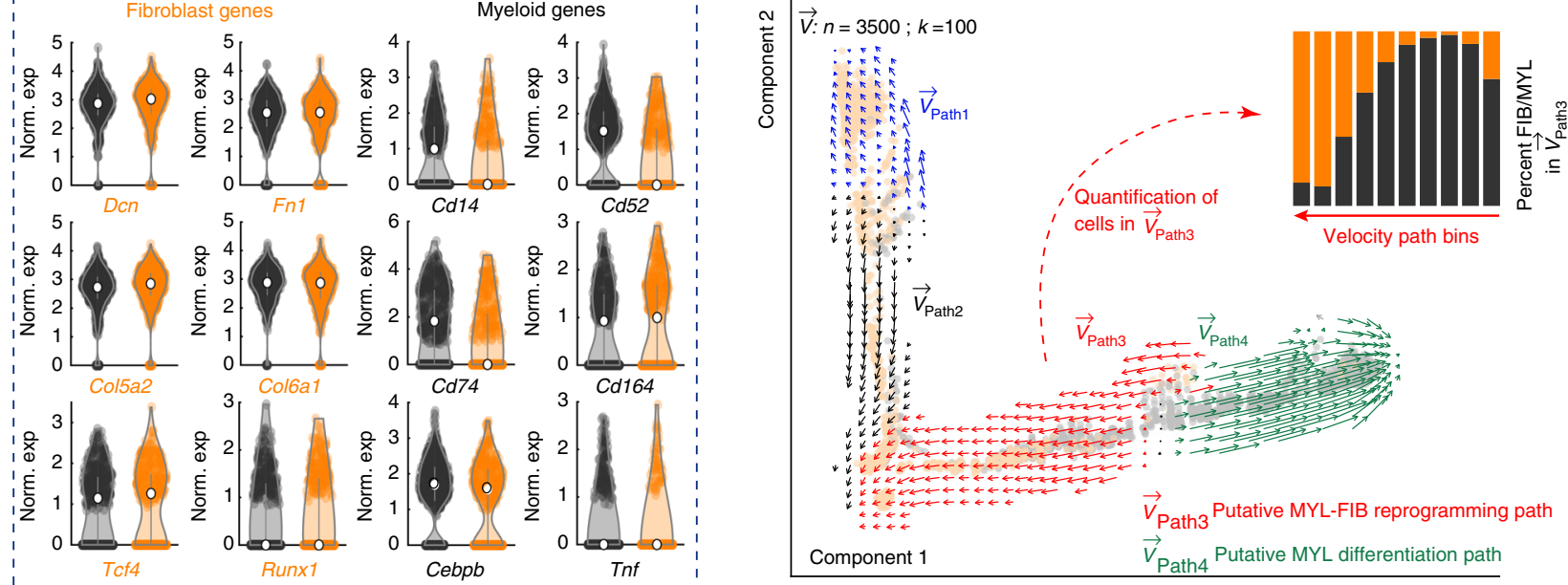

'Quantification
cells in $\vec{V}_{\text {Path3 }}$

Velocity path bins

Component 1
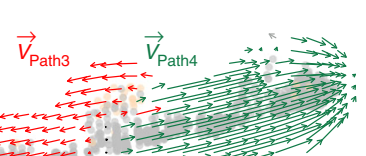

(2)
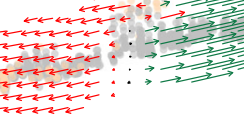

.

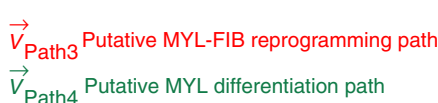

Next, we wounded BMT mice reconstituted with Sm22-Cre;

lineage constituted $\sim 30 \%$ at both 28 days ( $n=3$ biologically independent samples) and 2 months PW $(n=3$ biologically independent samples) (Fig. 6b-d). In contrast, BMT mice that received $\mathrm{GFP}^{+} \mathrm{CD}^{2} 5^{\text {neg }}$ non-hematopoietic $\mathrm{BM}$ fraction, which includes mesenchymal stromal cells, had no $\mathrm{GFP}^{+}$contribution to the wound (Fig. $7 \mathrm{~b})$ despite showing BM repopulation $(n=11$ animals; these BMT mice also received wild type whole BM support cells) (Supplementary Figure 18b).
$R 26 R$ HSCs. In this model, we expected lacZ expression to mark contractile cells that derived from reconstituted hematopoietic lineage cells (but not from transplanted HSCs directly), that activated $S m 22$ promoter-controlled Cre recombinase. Indeed, consistent with the possibility of long-lasting hematopoietic contribution to wound myofibroblasts we observed lacZ-positive cells in wounds from Sm22-Cre;R26R HSCs BMT mice $(n=9$ 
Fig. 4 Identification of rare myeloid-derived myofibroblasts in day 12 wounds. a Expression levels of myeloid marker Lyz2 shown as pseudotime feature plot. Cells with the highest expression level are colored black. $L y z 2^{+}$cells are present throughout the pseudotime. $\mathbf{b}$ Overlay of $L y z 2$ and contractile markers Acta2 and Tagln expression onto the t-SNE space reveals $\mathrm{Lyz2}^{+} / \mathrm{Acta2}^{+}$and $\mathrm{Lyz2}^{+} / \mathrm{Tag} / \mathrm{n}^{+}$double-positive wound fibroblasts. c Overlay of $\mathrm{Lyz2}$, Acta2, Tagln and fibroblast marker Col12a1 onto the t-SNE space reveals $\mathrm{Lyz}^{+} / \mathrm{Acta2}^{+} / \mathrm{Tag}_{\mathrm{I}} \mathrm{n}^{+} / \mathrm{Col} / 2 \mathrm{a} \mathrm{1}^{+}$quadruple-positive cells among wound fibroblasts. Quantification (bottom) shows that quadruple-positive cells are present in all wound fibroblast subclusters, with subclusters sC9 (pink) and sC10 (teal) being the most enriched. d Overlay of Acta2, Tagln and Col12a1 expression with that of the hematopoietic progenitor-associated marker Cd34 and myeloid

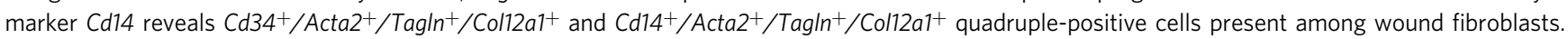
e Joint pseudotime ordering on $L y z 2^{+}$wound myeloid cells (black) and Lyz2+ fibroblasts (orange). Cells with normalized UMI ( $\mathrm{nUMI}$ ) counts $>0$ were selected for this analysis. $\mathbf{f}$ Stacked bar chart shows relative distribution of fibroblasts and myeloid cells across the pseudotime, which was divided into 10 equal bins. $\mathbf{g}$ Middle two bins show almost equal mixing of both cell types with hybrid expression patterns, simultaneously enriched for established fibroblast-specific and myeloid-specific genes. $\mathbf{h}$ Distribution of cells expressing contractile markers Acta2 and Tagln shown as pseudotime feature plots. Cells were considered positive if their nUMI counts were $>2$. Positive fibroblasts are colored orange, positive myeloid cell-black and negative cells - light gray. Positive cells are present across the entire pseudotime. $\mathbf{i}$ scEpath analysis performed on pseudotime from e along the fibroblast-to-myeloid trajectory identified five gene clusters ( $\mathrm{rC} 1$ through $\mathrm{rC5}$ ) of pseudotime-dependent genes. Heatmap is shown on the left. Average gene expression, gene counts and selected genes in all five clusters are shown on the right. $\mathbf{j}$ RNA velocity analysis distinguished several sets of velocity vectors across the pseudotime. Path3 vectors (red) encompass the middle bins from $\mathbf{g}$ and appear to represent putative transition of Lyz2+ myeloid cells into fibroblasts. Path4 vectors (green) appear to mark differentiation of wound-resident myeloid cells. FIB: fibroblast, MYL: myeloid cell

animals) 6 months after wounding (Fig. 6e-h). This was contrasted by the absence of lacZ-positive cells in the wounds of BMT mice reconstituted with Sm22-Cre;R26R CD45 neg BM cells ( $n=4$ animals) (Fig. 6i). We also found lacZ-expressing cells along many blood vessels (Fig. 6e, f), suggesting that upon BMT hematopoietic lineage cells may also participate in reconstituting perivascular mural cells.

Hematopoietic lineage cells convert to de novo adipocytes. Because fibroblast and fat lineages are closely related developmentally, we hypothesized that some hematopoietic lineage cells that initially convert into myofibroblasts might then become de novo adipocytes. Although contested ${ }^{42,43}$, several studies have reported that, in principle, myeloid cells can convert into adipocytes upon integration into pre-existing fat tissue ${ }^{44-46}$. Contribution of myeloid cells towards fat is variable and is dependent on gender and anatomical site, with visceral fat in female mice experiencing up to $10 \%$ contribution $^{44}$.

We employed both BMT and non-BMT mouse models to evaluate this possibility. Indeed, wounds in $\mathrm{GFP}^{+}$HSCs BMT mice contain many $\mathrm{GFP}^{+}$cells that co-stained for the adipocyte marker FABP4 in areas surrounding neogenic hair follicles (Fig. 7c). By taking advantage of the fact that in mice the expression of Retn, which encodes for Resistin, is exclusive to mature fat cells, we generated Retn-lacZ HSCs BMT and RetnlacZ CD45 ${ }^{\text {neg }}$ BMT models using the approach described above (Fig. 6a). In these mice, nuclear lacZ expression can be used to genetically identify functionally mature de novo adipocytes that may have derived from transplanted BM progenitors rather than from the host sources. We consistently observed formation of occasional lacZ-positive de novo adipocytes in the hair-bearing wounds of Retn-lacZ HSCs BMT mice ( $n=72$ animals) (Fig. $7 \mathrm{f}$, g), but not in Retn-lacZ CD45 ${ }^{\text {neg }}$ BMT mice ( $n=50$ animals) (Fig. $7 d$ ). Importantly, in agreement with a previous report ${ }^{44}$, we also observed integration of hematopoietic-derived lacZ-expressing adipocytes into normal fat of Retn-lacZ HSCs BMT mice (Fig. 7e).

Next, we examined if the hematopoietic lineage in BMT mice contributes to de novo adipocytes via direct conversion rather than cell fusion. For that purpose, we generated BMT models in which we transplanted $\mathrm{GFP}^{+}$HSCs into $\mathrm{RFP}^{+}$hosts and $R 26 R^{+/-}$HSCs into Fabp4-Cre hosts. In the first model, all fused cells can be identified on the basis of GFP/RFP double fluorescence. In the second model only fusion-derived de novo adipocytes contain Fabp4-Cre construct necessary for R26R reporter activation. We observed only very occasional GFP/RFP double positive cells in the hair-bearing wounds of $\mathrm{GFP}^{+}$HSCs into $\mathrm{RFP}^{+} \mathrm{BMT}$ mice ( $n=8$ animals) (Fig. 7h). LacZ-positive adipocytes were not observed in regenerated wounds and in normal fat of $R 26 R^{+/-}$ HSCs into Fabp4-Cre BMT mice ( $n=13$ animals) (Fig. 7i), in contrast to robust lacZ expression in fat of non-BMT Fabp4-Cre; $R 26 R$ mice (Fig. 7j). These experiments confirm that hematopoieticto-adipose lineage conversion, rather than cell fusion, takes place during de novo fat regeneration in the wound.

Lineage study confirms myeloid origin of de novo adipocytes. We then studied wound healing in Cd45-Cre;R26R mice to verify that hematopoietic cells contribute to de novo fat physiologically and not only in the context of BMT models. In these mice, where Cre activity is restricted to the hematopoietic lineage ${ }^{47}$, we observed consistent, albeit occasional formation of lacZ-positive de novo adipocytes ( $n=9$ animals) (Fig. 8a; Supplementary Figure 20a). Similarly, lacZ-positive adipocytes formed in the wounds of LysM-Cre;R26R mice ( $n=12$ animals) (Fig. 8b, c; Supplementary Figure 20b), and a portion of tdTomato ${ }^{+}$cells, derived from 26 days PW hair-bearing wounds of LysM-Cre; tdTomato mice, differentiated into lipid-laden adipocytes when cultured under adipogenic conditions ( $n=3$ replicate cultures) (Fig. 8d). This suggests that hematopoietic lineage contribution to fat neogenesis is mediated via myeloid progenitors. Consistent with occasional distribution patterns of lacZ-positive adipocytes in wounds of LysM-Cre;R26R mice, LysM-Cre;Ppary ${ }^{-1-}$ mutants did not have a clear de novo fat defect $(n=20$ animals) (Fig. $8 \mathrm{e}$ vs. 8f), characteristic of Sm22-Cre;Ppary ${ }^{-1-}$ mice described in Plikus, Guerrero-Juarez ${ }^{5}$. Curiously, in both mouse models, we also observed occasional formation of neogenic hair follicles with lacZ-positive DPs and dermal sheath (Fig. 8a, b, red arrowheads; Supplementary Figure 20), suggesting that the lineage plasticity repertoire of myeloid cells during wound regeneration might extend beyond adipogenesis. Taken together, our lineage tracing studies help to establish the role of myeloid cells as one of the sources of adipogenic progenitors during wound regeneration.

\section{Discussion}

Traditionally, adult mammals are considered to have limited regenerative abilities and scarring is thought to be the default repair response. The notable exceptions to this rule are digit tip regeneration after amputation ${ }^{48,49}$ and neogenesis of hair follicles ${ }^{4,6,7,14}$ and $\mathrm{fat}^{5}$ in the center of large excisional wounds. Intriguingly, lineage studies reveal important differences in the regenerative strategies between these two systems. Epithelial and 
a Validation of $3^{\prime}$-end single cell transcriptomic data
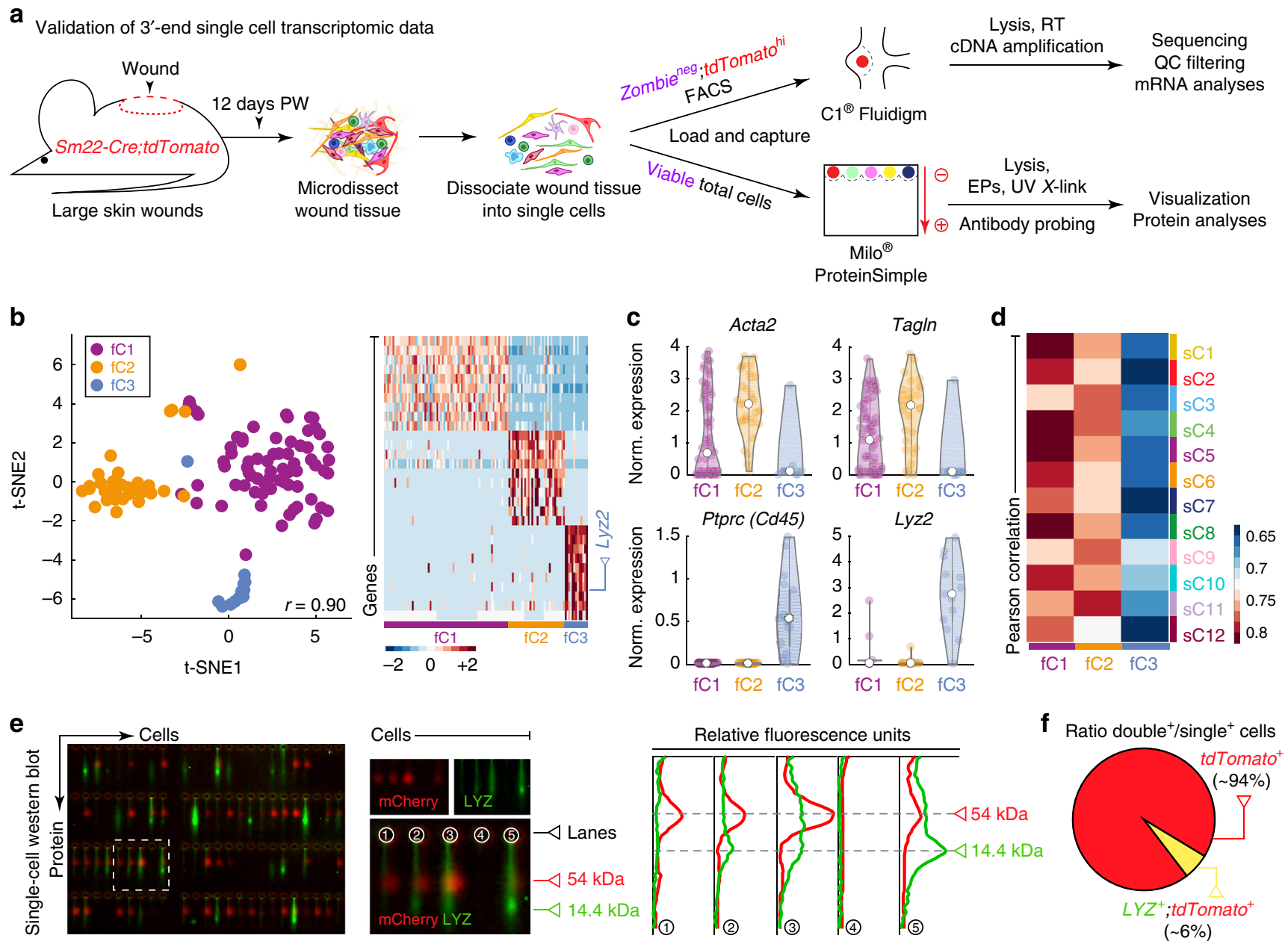

g PDGFRA LYZDAPI

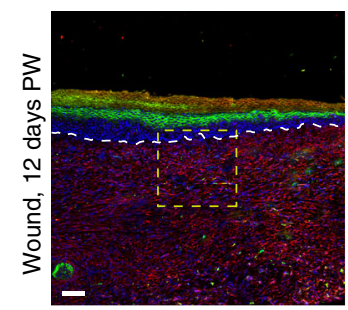

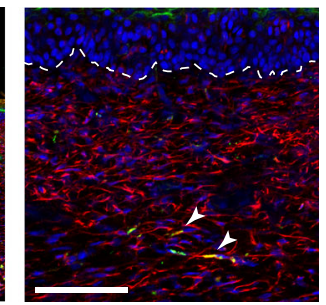

h
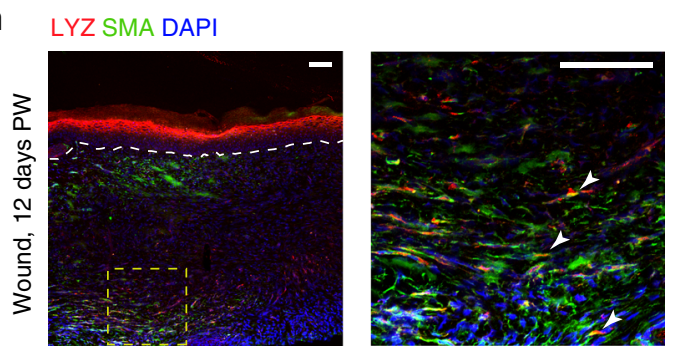

Fig. 5 Validation of myeloid-derived myofibroblasts in day 12 wounds. a Schematic of cell isolation from day 12 wounds in Sm22-Cre;tdTomato mice, sorting for tdTomato ${ }^{+}$cells, capture by microfluidic device, sequencing and downstream analysis, and wound cell processing for single-cell western blot. $\mathbf{b}$ t-SNE plot revealed heterogeneity among tdTomato ${ }^{+}$wound cells. 116 sequenced cells were analyzed and three clusters were identified. Heatmap of top differentially expressed genes is shown on the right and Lyz2 is marked. c Violin plots of contractile markers Acta2 and Tagln, pan-hematopoietic marker Ptprc (aka Cd45) and myeloid marker Lyz2. d Pearson correlation analysis between day 12 tdTomato+ cell clusters and day 12 wound fibroblasts subclusters from Fig. 2a. e Single-cell western blot on unsorted cells from day $12 \mathrm{Sm} 22$-Cre;tdTomato wounds revealed LYZ-expressing myofibroblasts. Chips were costained for LYZ (green) and tdTomato (mCherry, red). Graph on the right shows LYZ+/tdTomato ${ }^{+}$double-positive cells in lanes \#2, \#3, and \#5. Cell in lane \#1 is single-positive for tdTomato and cell in lane \#4 is double-negative. $\mathbf{f}$ Quantification shows that $\sim 6 \%$ of tdTomato ${ }^{+}$wound cells co-express LYZ (77 out of 1293 cells). See also Supplementary Figure 14. g Co-staining of day 12 wounds for LYZ (green) and PDGFRA (red) identified occasional doublepositive fibroblasts. h Co-staining of day 12 wounds for LYZ (red) and SMA (green) identified occasional double-positive myofibroblasts. Immunostaining images shown on $\mathbf{g}$ and $\mathbf{h}$ are representative of the marker staining patterns observed in three or more independently stained samples. PW: post-wounding, FACS: fluorescence-activated cell sorting, RT: reverse transcription, EP: electrophoresis, QC: quality control. Size bars: $\mathbf{g}, \mathbf{h}-100 \mu \mathrm{m}$

mesenchymal structures in the digit tip regenerate from several types of fate-restricted progenitors and no multipotent progenitors or lineage reprogramming events are observed ${ }^{48,49}$. In contrast, large skin wounds demonstrate broadened lineage plasticity. Although progeny of preexisting hair-fated bulge stem cells migrate into wound epidermis, they do not partake in hair follicle neogenesis ${ }^{4}$. Instead, new hair follicles regenerate from non-bulge epithelial stem cells ${ }^{15,20}$, among other sources. Fat neogenesis is driven by lineage reprogramming of non-adipogenic wound myofibroblasts 5 . DP neogenesis also likely relies on myofibroblast reprogramming strategy ${ }^{14}$.

Are all wound myofibroblasts identical or heterogeneous in terms of their origin, properties, and morphogenetic competence? While studying cell origin requires genetic fate mapping, and 
a

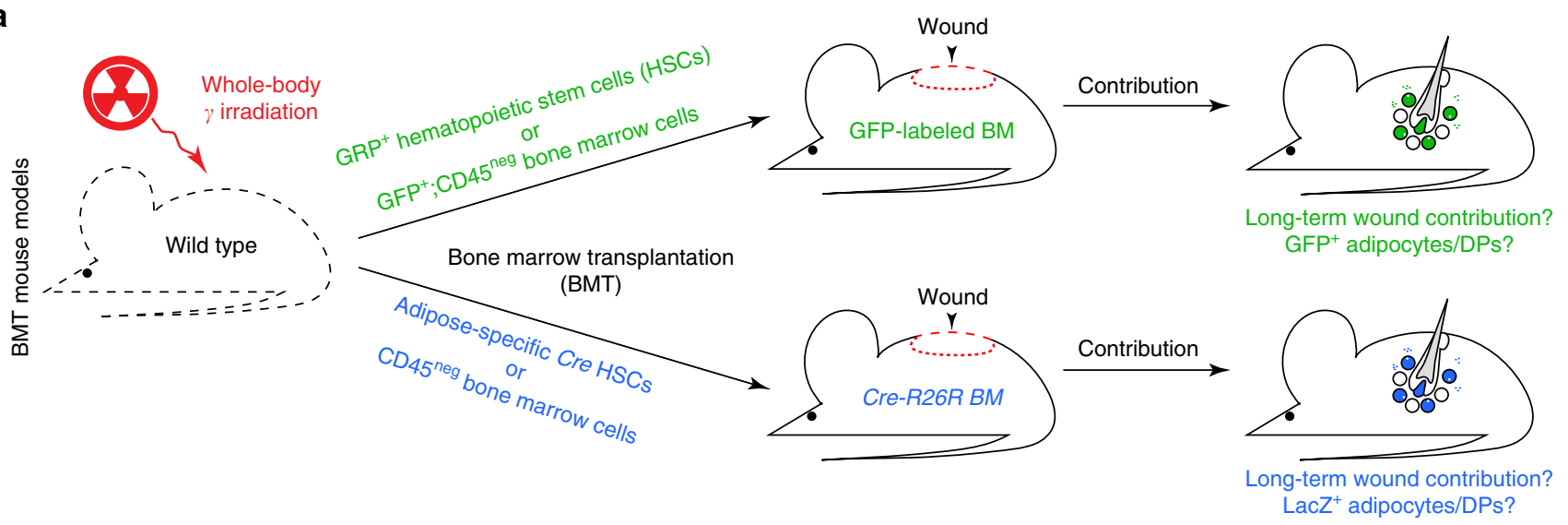

b GFP $^{+}$HSCs BMT, 28 days PW C
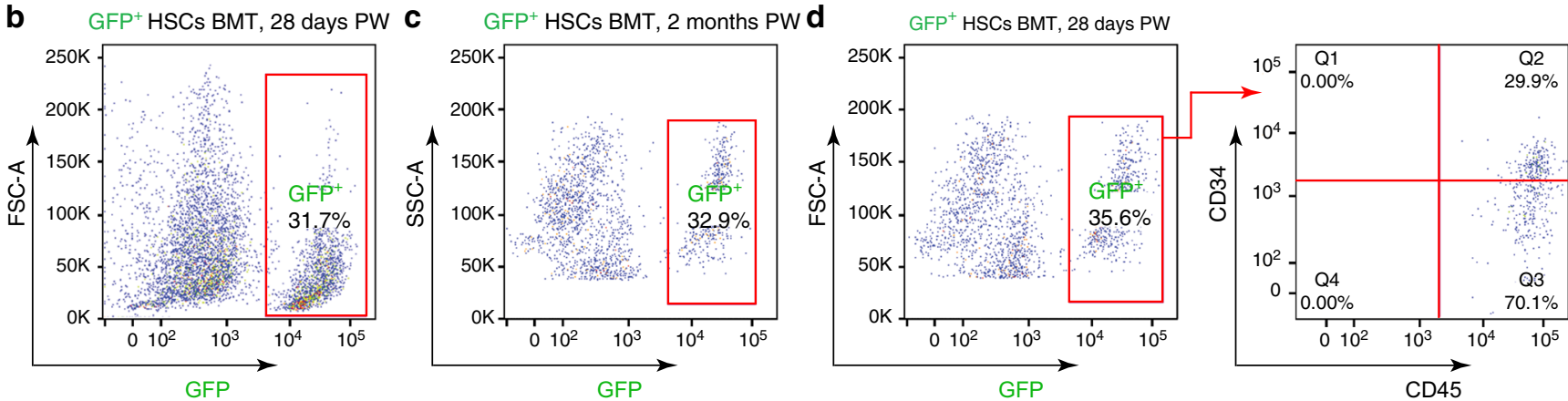

g Sm22-Cre;R26R HSCs BMT

e Sm22-Cre; R26R HSCs BMT Normal skin

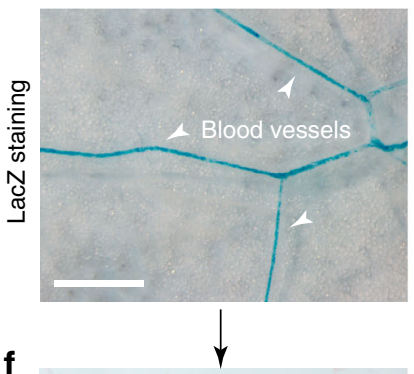
Wound, 6 months PW

Significant contribution by Sm22-Cre ${ }^{+}$hematopoietic cells
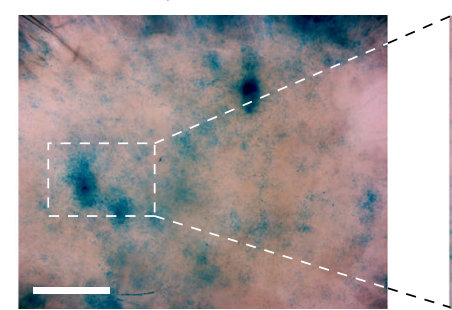

i Sm22-Cre;R26R CD45 ${ }^{\text {neg }}$ BMT Wound, 6 months PW
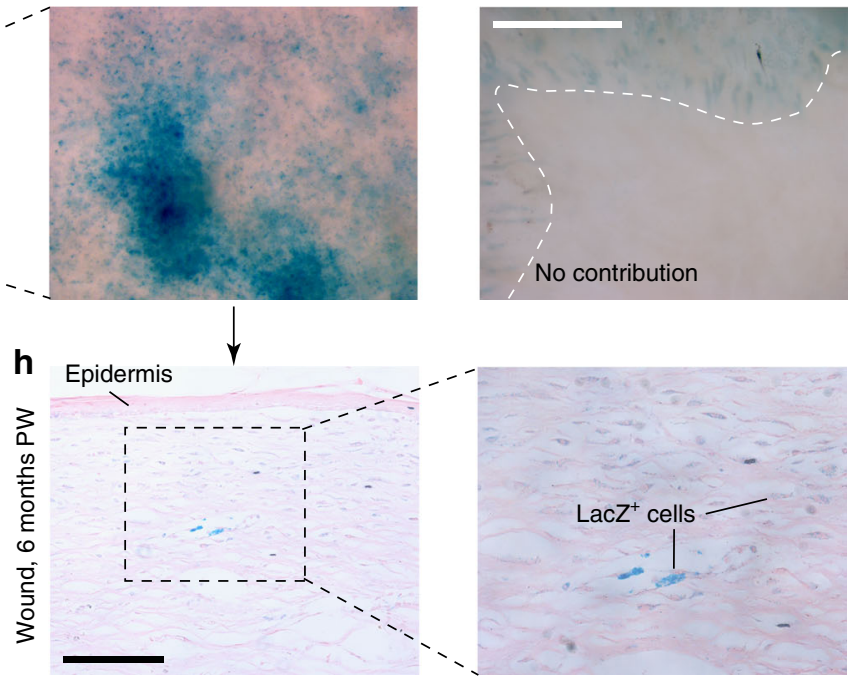

Fig. 6 Hematopoietic lineage contributes toward regenerating wounds in BMT mice. a Schematic representation of the key BMT experiments used to assess the contribution of hematopoietic lineage cells to de novo adipocytes. b-d Based on flow cytometry analysis, the hematopoietic lineage contributes $\sim 30 \%$ of the cells in dermal wound tissue 28 days and 2 months PW in GFP+ HSCs BMT mice. e-h LacZ expression patterns in the wounds of Sm22-Cre; R26R HSCs BMT mice confirmed that a portion of contractile wound cells $(\mathbf{g}, \mathbf{h})$ and perivascular contractile cells (arrowheads on $\mathbf{e}$ ) originate from the hematopoietic lineage. i Wounding experiments in Sm22-Cre;R26R CD45 neg BMT mice showed that non-hematopoietic BM cells do not contribute toward contractile wound and peri-wound cells. Images shown in e-i are representative of the lacZ expression patterns observed in three or more independent samples. HSC: hematopoietic stem cell, BM: bone marrow, BMT: bone marrow transplantation, DP: dermal papilla, PW: post-wounding day. Size bars: e, $\mathbf{g}, \mathbf{i}-1 \mathrm{~mm} ; \mathbf{f}, \mathbf{h}-125 \mu \mathrm{m}$

assessment of morphogenetic competence requires functional studies, scRNA-seq allows large-scale profiling of cell properties in complex tissues. Recently, this approach has been applied to studying cellular heterogeneity in the skin, including epithelial cells of mouse hair follicles 50,51 , and early wounds ${ }^{52}$, and keratinocytes $^{53}$ and fibroblasts ${ }^{54,55}$ of human skin. Tabib, Morse $^{55}$ identified two major populations of human skin fibroblasts, characterized by co-expression of $S F R P 2^{+} / D P P 4^{+}$and $\mathrm{FMO}^{+} / \mathrm{LSP} 1^{+}$markers. These further subdivide into several subpopulations. Philippeos, Telerman ${ }^{54}$ identified five fibroblast 

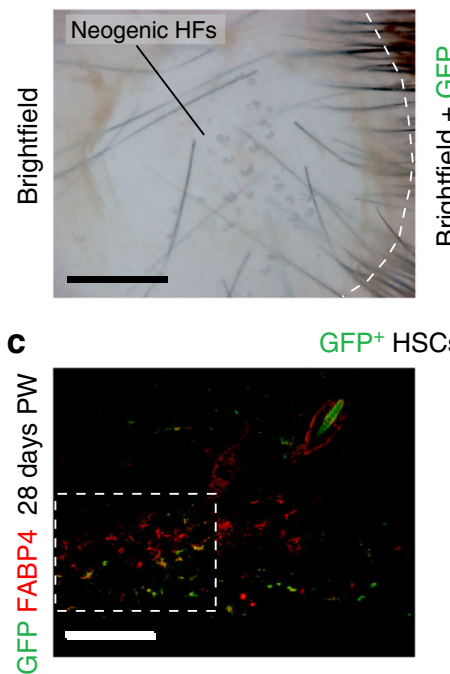

e $\quad$ Retn $^{+/-}$HSCs BMT $\rightarrow$ WT $\quad$ f
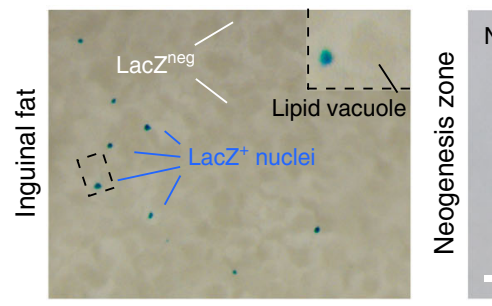

b

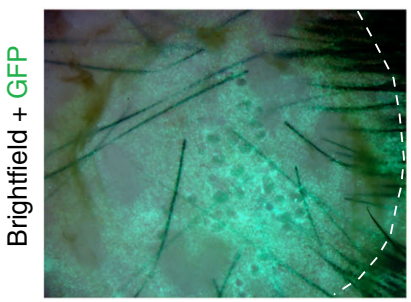

$\mathrm{GFP}^{+} \mathrm{CD}^{\text {neg }} \mathrm{BMT}, 28$ days PW
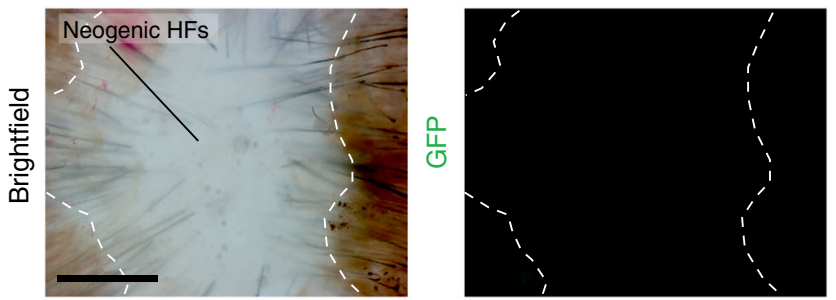

d $\quad$ Retn $^{+/-}$CD45 ${ }^{\text {neg }} \mathrm{BMT} \rightarrow \mathrm{WT}, 28$ days PW
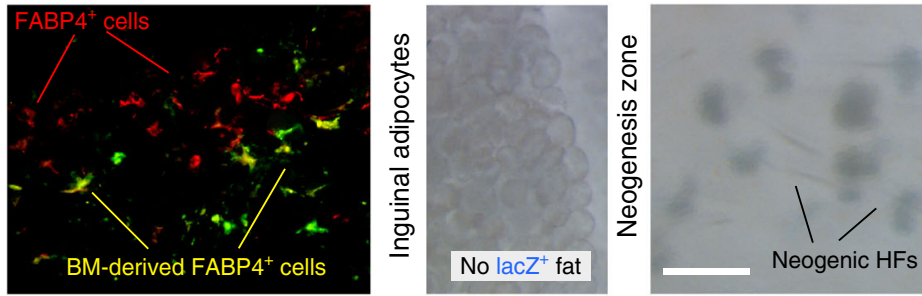

No lac $Z^{+}$fat h

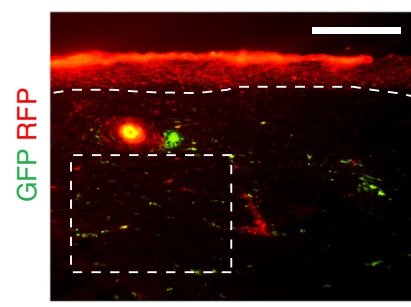

Retn $^{+/-}$HSCs BMT $\rightarrow$ WT, 28 days PW

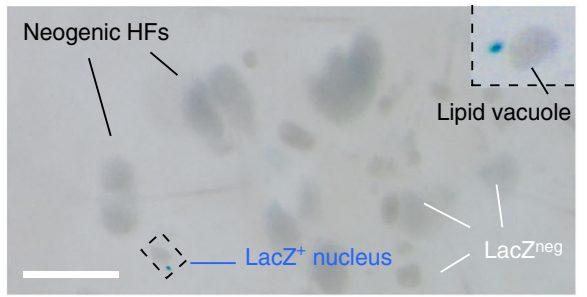

\section{g}

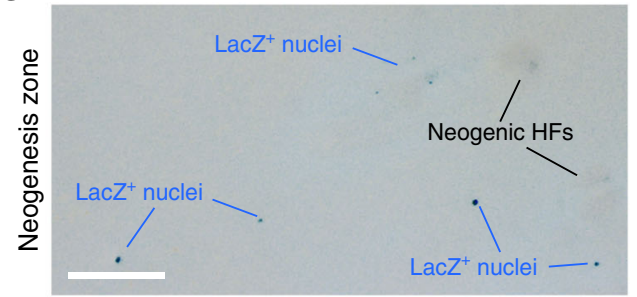

Fusion test: $\mathrm{GFP}^{+} \mathrm{HSC}$ BMT $\rightarrow$ RFP, 28 days PW

i Fusion test: $R 26 R \rightarrow$ Fabp4-Cre, 28 days PW

j Fabp4-Cre;R26R
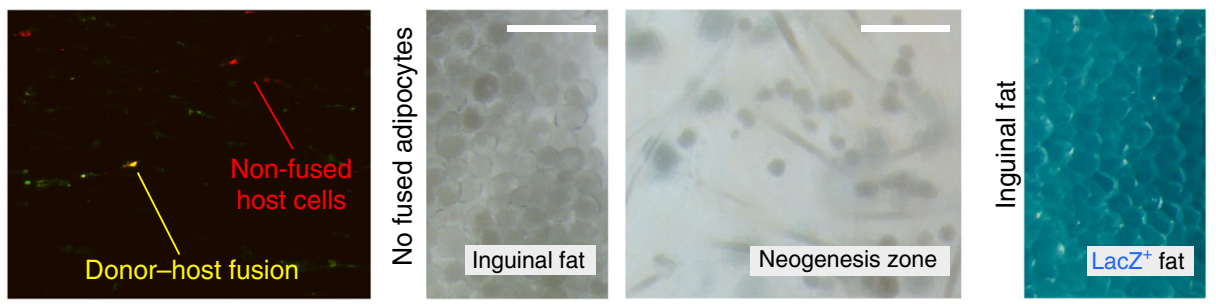

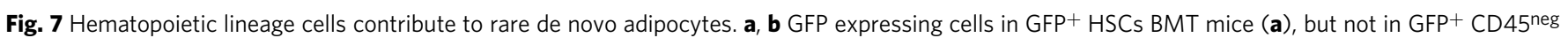

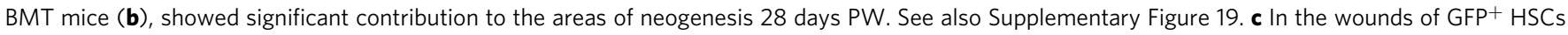
BMT mice, GFP+ cells localize around neogenic hair follicles and a portion of them co-expresses FABP4 at 28 days PW. Image is representative of the marker staining pattern observed in three or more independently stained samples. $\mathbf{d}$ CD45neg BM cells do not contribute toward adipocytes in the inguinal fat or regenerated 28 days PW wounds of BMT mice reconstituted with Retn-lacZ CD45 ${ }^{\text {neg }}$ cells. e- $\mathbf{g}$ In contrast, hematopoietic lineage cells consistently contributed toward rare adipocytes (cells with nuclear lacZ expression) in the inguinal fat (e) and regenerated 28 days PW wounds (f, $\mathbf{g}$ ) of BMT mice reconstituted with Retn-lacZ HSCs. Insets on e and $\mathbf{f}$ show positive adipocytes with lipid vacuole and peripherally-positioned lacZ+ nucleus. $\mathbf{h}$ Only rare cell fusion events were identified on the basis of GFP/RFP double fluorescence in 28 days PW wounds of GFP + HSCs into RFP + BMT mice. Fused and non-

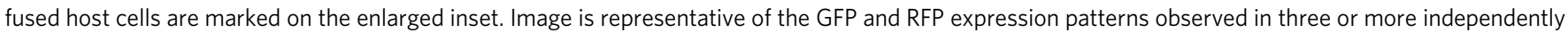
stained samples. $\mathbf{i}$, j Lack of cell fusion-dependent de novo adipocytes in wounds of BMT models was confirmed by the absence of lacZ+ cells in R26R HSCs into Fabp4-Cre BMT mice (i). This was contrasted by robust lacZ expression in the inguinal fat of Fabp4-Cre;R26R mice (j). PW: post-wounding, BM: bone marrow, BMT: bone marrow transplantation, HSC: hematopoietic stem cell, HF: hair follicle. Size bars: $\mathbf{a}, \mathbf{b}-1 \mathrm{~mm} ; \mathbf{c}, \mathbf{h}-50 \mu \mathrm{m} ; \mathbf{d}, \mathbf{f}, \mathbf{g}, \mathbf{i}-200 \mu \mathrm{m}$

populations: upper and lower dermal fibroblasts, pericytes, and two uncharacterized populations. scRNA-seq has also been used to study heterogeneity of disease-associated fibroblasts in the synovial tissue upon rheumatoid arthritis ${ }^{56,57}$ and in the lung upon bleomycin-induced fibrosis ${ }^{58}$.

Here, we studied fibroblast heterogeneity in the mouse model for wound-induced regeneration at 12 days PW when wound reepithelialization is completed and preceding hair follicle neogenesis. At later time points 15 days and 21 days PW, we anticipated a decrease in SMA expression and change in fibroblast types. We show that wound fibroblasts can be broadly classified into two major populations on the basis of their transcription factor signatures and PDGF receptor expression patterns. Pdgfrahigh fibroblasts, which dominate in the wound, further subdivided into upper and lower dermal fractions, based on their localization and gene expression. Pdgfralow cells constituted nearly a quarter of all wound fibroblasts and localized throughout wound dermis. Lineage tracing will be required to determine which fibroblast population preferentially contributes toward newly regenerating hair follicle mesenchyme and adipocytes. Prominent additional heterogeneity exists within both populations, and pseudotime and RNA velocity analyses predict 
a

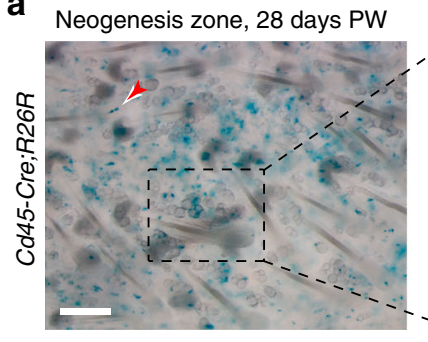

C

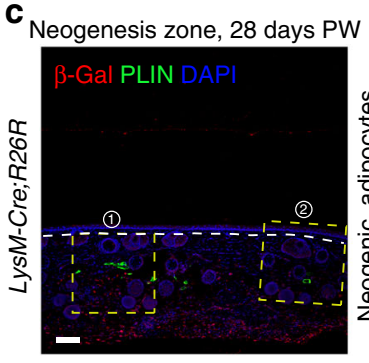

e

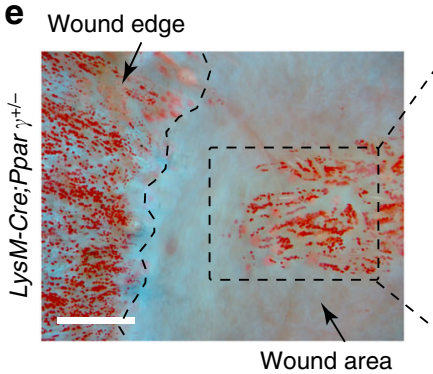

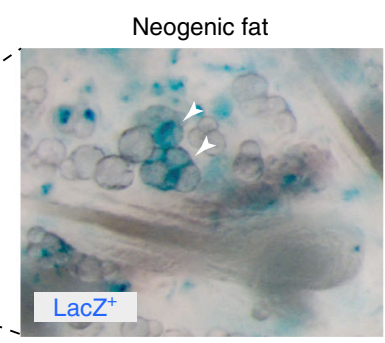

b
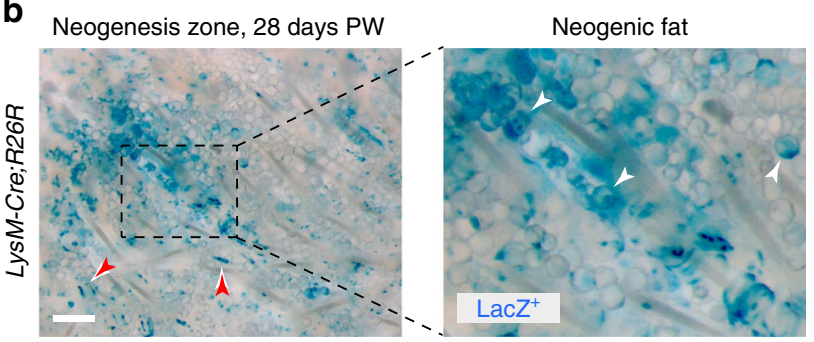

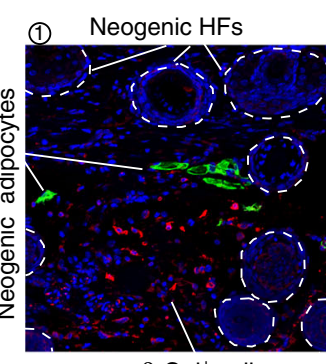

$\beta-\mathrm{Gal}^{+}$cells

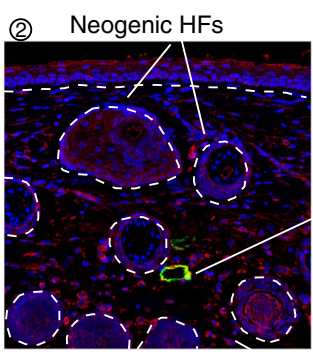

$\beta-\mathrm{Gal}^{+}$cells

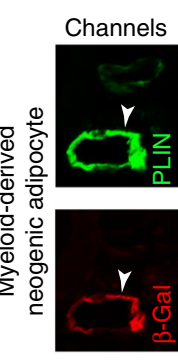

$\mathbf{f}$

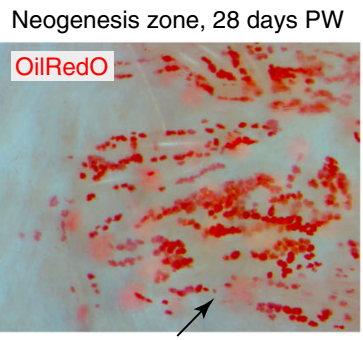

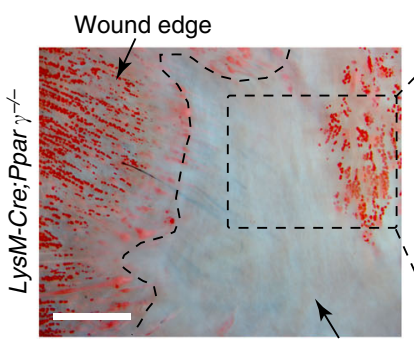

d

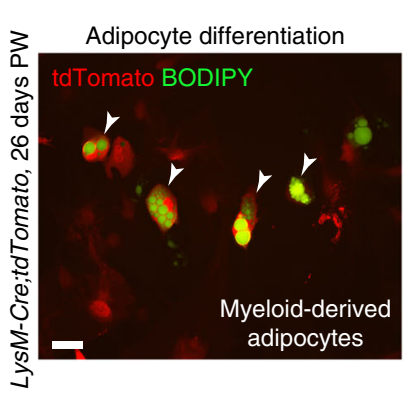

Fig. 8 Myeloid lineage cells contribute to rare de novo adipocytes. $\mathbf{a}, \mathbf{b}$ LacZ $^{+}$clusters of de novo adipocytes (white arrowheads) were consistently identified in the wounds of pan-hematopoietic specific Cd45-Cre;R26R (a) and myeloid-specific LysM-Cre;R26R mice (b). Red arrowheads mark lacZ+ DPs of neogenic hair follicles. See also Supplementary Figure 20. c Co-staining of day 28 LysM-Cre;R26R wounds for $\beta$-Gal (red) and PLIN (green) identified both single-positive (inset 1) and double-positive mature adipocytes (inset 2) in the region of neogenesis. Image is representative of the marker staining patterns observed in three or more independently stained samples. d Lipid-laden adipocytes (BODIPY+, green) can be differentiated in vitro under adipogenic conditions from tdTomato ${ }^{+}$cells (red), isolated from day 26 hair-bearing wounds from LysM-Cre;tdTomato mice. e, $\mathbf{f}$ In agreement with the limited lineage contribution from myeloid cells toward de novo adipocytes LysM-Cre;Ppar ${ }^{-/-}$mutants regenerated nearly normal-looking de novo fat (compare mutant wound on $\mathbf{f}$ with heterozygous control on e). Adipocytes were stained red with OilRedO. PW: post-wounding, HF: hair follicle. Size bars: a, b-200 $\mu \mathrm{m} ; \mathbf{c}-100 \mu \mathrm{m} ; \mathbf{d}-25 \mu \mathrm{m} ; \mathbf{e}, \mathbf{f}-1 \mathrm{~mm}$

three distinct fibroblast-to-myofibroblast differentiation trajectories. Future work should aim to understand the functional significance of this heterogeneity in the context of regeneration and to lineage trace wound fibroblast origin to distinct skin fibroblasts in the unwounded skin.

BM-derived progenitors, including myeloid cells, endothelial progenitors, and circulating mesenchymal stem cells can contribute new stromal cells toward injured tissues in various organs. Scar tissue in heart following myocardial infarction ${ }^{59}$, cornea following keratectomy ${ }^{60}$, and lung in pulmonary fibrosis ${ }^{61}$ contains BM-derived collagen-producing myofibroblasts. In skin, studies document $\mathrm{BM}$ giving rise to fibroblasts at the injury sites $^{35-41}$, while some studies report this contribution to be minimal ${ }^{62,63}$. Such a discrepancy is likely attributed to several factors, including the type and extent of injury and experimental timing. The contribution from BM progenitors toward repairing tissues was shown to increase with the extent of injury $37,64,65$, yet most of the previous studies were performed on small wounds. Also, many previous studies failed to evaluate long-term $\mathrm{BM}$ contribution, yet BM-derived fibroblasts in the scar were shown to peak after 22 days $^{35}$.
Our data from large excisional wounds shows that the contribution from myeloid cells to wound fibroblasts is small yet significant, between $6 \%$ and $11.3 \%$, depending on the assessment method. scRNA-seq analysis revealed an intermediate state of wound cells, displaying dual gene expression signature, enriched for contractile and mesenchymal extracellular matrix genes, as well as myeloid cell surface antigens and inflammatory cytokines. RNA velocity analysis showed that wound cells progress through this intermediate state in a myeloid-to-fibroblast direction. We also showed that at least a portion of these cells can convert into de novo adipocytes around neogenic hair follicles. Previously, adipogenic conversion of myeloid cells has been shown both in vitro 66,67 and in vivo in major adipose depots, such as in inguinal fat ${ }^{44,45}$; although some studies contested these observations ${ }^{42,43}$. Most recently, $\sim 10 \%$ of adipocytes were shown to form from hematopoietic lineage in human subjects undergoing BMT treatment ${ }^{46}$, while in another human BMT study, up to $35 \%$ of adipocytes were traced to transplanted BM sources ${ }^{45}$.

Overall, our findings illustrate the dynamic nature of fibroblast identities during wound healing, and the powerful woundinduced plasticity of myeloid lineage cells. scRNA-seq analysis 
infers several pathways fibroblasts follow during wound healing, including contractile and regenerative. The lineage relationship between myeloid cells and adipogenesis suggests further characterization of the factors influencing plasticity and fate switching could uncover potential therapeutic approaches to treating wounds and scars.

\section{Methods}

Mouse models. The following mouse models were used in this study: C57BL/6J (JAX stock 000664), Retn-lacZ, Sm22-Cre (JAX stock 004746), Cd45-Cre, LysM-Cre (JAX stock 004781), Fabp4-Cre (JAX stock 005069), Ppary flox (JAX stock 004584), R26R (JAX stock 003474), tdTomato (JAX stock 007909), GFP (JAX stock 004353), RFP (JAX stock 006051), and Rag1-/- (JAX stock 002216).

Wounding experiments. All animal experiments were carried out in accordance with the guidelines of the Institutional Animal Care and Use Committee of the University of Pennsylvania and University of California, Irvine. Animals were anesthetized with isoflurane, hairs were clipped, skin site was disinfected and a single full thickness excisional wound was created on their dorsum using scissors (squared $s=1.5 \mathrm{~cm})^{4-6}$. Both male and female mice between 6 and 8 weeks of age were used. Following wounding, all animals were housed individually. Wounds were let to heal by secondary intention. No wound dressing was applied. Animals were used as biological replicates.

Bone marrow transplantation. Bone marrow cells were flushed from the long bones with Dulbecco's Modified Eagle's Medium supplemented with 5\% heatinactivated calf serum (Gibco). To obtain a single cell suspension, cells were filtered through a $45 \mu \mathrm{m}$ nylon screen. Cells were blocked with anti-rat and anti-mouse IgG (Sigma) for $15 \mathrm{~min}$. For isolation of HSCs, cells were then stained with CD45 (APC-Cy7, BD Pharmingen), CD150 (APC, Biolegend), CD48 (Pacific Blue, Biolegend), SCA1 (PE-Cy5.5, Invitrogen), and c-KIT (PE-Cy7, eBioscience), as well as $\mathrm{PE}$-conjugated lineage markers including CD19, B220, CD3, CD4, CD8, GR-1, MAC-1, NK1.1, and TER119 (eBioscience). Cells were resuspended in DAPI to discriminate live from dead cells. HSCs were sorted by isolating Lineage ${ }^{\text {neg }}, \mathrm{SCA}^{+}{ }^{+}$, c-KIT ${ }^{+}, \mathrm{CD}_{150}+, \mathrm{CD} 48^{\text {neg }}$ cells using a BD FACSAria II flow cytometer (BD Biosciences). Approximately 150,000-2,000,000 CD45 ${ }^{+}$cells or 2300-4400 HSCs, or $\sim 150,000-2,000,000$ CD $45^{\text {neg }}$ cells were resuspended in phosphate buffered saline with 100,000-200,000 whole bone marrow cells for reconstitution and injected into lethally irradiated isogenic wild type host mice. For Sm22-Cre;R26R BMT experiments, cells were transplanted into immunodeficient $R a g 1^{-/}$mice. Donor chimerism was assessed by measuring GFP or CD45.1 vs. CD45.2 levels by FACS in the peripheral blood 8 weeks after transplantation.

Whole mount lacZ staining. To detect lacZ activity, freshly isolated wound tissue samples were incubated with X-gal reagent in lacZ staining buffer ${ }^{4,5}$. Samples were post-fixed in $4 \%$ PFA.

Whole mount OilRedO staining. PFA-fixed wound tissue samples were preincubated in propylene glycol and then stained with OilRedO buffer for $20 \mathrm{~min}$. Samples were then washed in propylene glycol and stored in $0.05 \%$ aqueous solution of sodium azide.

Histology and immunohistochemistry. Tissues were fixed in 4\% PFA, dehydrated, paraffin embedded, and sectioned at $7-12 \mu \mathrm{m}$ thickness. Frozen tissues were sectioned at $12 \mu \mathrm{m}$ thickness. Immunostaining was performed both on frozen and paraffin sections. Heat-based antigen retrieval was performed when necessary. Tissue sections were blocked in either 3\% BSA, 3\% Donkey serum, or 3\% mouse IgG from Mouse on Mouse (MOM) kit (Vector Labs). The primary antibodies used were goat anti-FABP4 (1:200; R\&D Systems), mouse anti-SMA (1:500; R\&D Systems), rabbit anti-RGS5 (1:250; Proteintech), rabbit anti-LYZ (1:250; Abcam), rabbit anti-CRABP1 (1:800; Cell Signaling), goat anti-CYP26B1 (1:50; Novus Biologicals); rabbit anti-G0S2 (1:100; Proteintech), mouse anti-MEST (1:250; Proteintech), goat anti-PDGFRA (1:350; R\&D Systems), chicken anti- $\beta$-Gal (1:500; Abcam), and rabbit anti-PLIN (1:800; Cell Signaling). Tissue sections were visualized with Nikon T inverted (Nikon) and Olympus FluoView confocal (Olympus) microscopes.

Cell culture and adipocyte differentiation. Primary cells were isolated from 26 days PW pooled wound tissues of LysM-Cre;tdTomato mice. Tissues were micro-dissected, minced, and incubated in a Collagenase IV solution for $60 \mathrm{~min}$ with constant rotation. Post-incubation, cells were filtered through a $40 \mu \mathrm{m}$ nylon screen, centrifuged, washed, resuspended in DMEM supplemented with $10 \%$ FBS, antibiotics, and antifungals and cultured at $37^{\circ} \mathrm{C}$ in a water-jacketed incubator with $5 \% \mathrm{CO}_{2}$ output. Once cells reached confluency, they were switched to adipocyte differentiation media (Cell Solutions) for $72 \mathrm{~h}$, upon which they were switched to adipocyte maintenance media (Cell Solutions) for additional 7 days.
Cells were incubated with BODIPY (Thermo) as per manufacturer's directions and visualized using a Nikon $\mathrm{T}$ inverted (Nikon) microscope.

$3^{\prime}$-end single cell RNA-sequencing. Pooled wound tissues ( $n=12$ animals) were collected from Sm22-Cre;tdTomato mice on 12 days PW. Tissues were micro-dissected, minced, and incubated in a Dispase II/Collagenase IV/Liberase solution for $60 \mathrm{~min}$ with constant rotation. Post-incubation, cell aggregates were mechanically dissociated using GentleMACS (MACS). Single cell suspensions were treated with $1 \mathrm{X}$ RBC lysis buffer, washed, and re-suspended in $0.04 \%$ UltraPure BSA (Biolegend). Dead cells were removed using the MS columns of the Dead Cell Removal Kit (MACS) as per manufacturer's directions. Live cells were resuspended in $0.04 \%$ UltraPure BSA and counted using the automated cell counter Countess (Thermo). GEM generation, barcoding, post GEM-RT cleanup, cDNA amplification, and cDNA library construction were performed using Single Cell 3' v2 chemistry (10X Genomics). Library quality control metrics were as follows: $550.19 \mathrm{pg} / \mu \mathrm{l}$ with an average size $\sim 454 \mathrm{bps}$. Libraries were sequenced on an Illumina HiSeq4000 platform (Illumina) (one lane, $100 \mathrm{PE}$ ). Cell counting, suspension, GEM generation, barcoding, post GEM-RT cleanup, cDNA amplification, library preparation, quality control, and sequencing were performed at the Genomics High Throughput Sequencing facility at the University of California, Irvine.

Data processing for $\mathbf{3}^{\prime}$-end transcripts. Transcripts were mapped to the $\mathrm{mm} 10$ reference genome (GRCm38.91) using Cell Ranger Version 2.1.0. The web summary of Cell Ranger statistics on cells, sequencing and mapping metrics prequality control assessment for downstream bioinformatics analyses were as follows -cell metrics: $\sim 22,322$ estimated number of cells, $\sim 84.2 \%$ fraction reads in cells, $\sim 13,819$ mean reads per cell, $\sim 1101$ median genes per cell, $\sim 19,070$ total genes detected, $\sim 2448$ median UMI counts per cell; sequencing metrics: $\sim 308,471,010$ total number of reads, $\sim 98.5 \%$ valid barcodes; mapping metrics: $\sim 90.4 \%$ reads mapped to genome, $\sim 85.5 \%$ reads mapped confidently to genome, $\sim 65.9 \%$ reads mapped confidently to transcriptome.

Quality control metrics for $\mathbf{3}^{\prime}$-end transcripts. For downstream analyses after initial Cell Ranger metric assessment, low-quality cells were removed to eliminate cell-specific biases. Quality control metrics included keeping cells displaying $<8000$ $\mathrm{UMI} /$ cell and $<2500$ genes/cell, and no more than $8 \%$ mitochondrial gene expression. Post-quality control, 21,819 cells remained for downstream bioinformatic analyses.

Downstream analyses of $\mathbf{3}^{\prime}$-end transcripts. Clustering of cells was performed using the Seurat $\mathrm{R}$ package ${ }^{25}$. In brief, digital gene expression matrices were column-normalized and log-transformed. To identify cell clusters, principle component analysis (PCA) was first performed on the list of highly variable genes. A set of highly variable genes was then identified by binning the average expression of all genes into evenly sized groups and computing the median dispersion (variance divided by the mean) in each bin. Genes were selected for inclusion in PCA with an average expression $>0.01$ and dispersion $>1.0$. To identify significant PCs, we used the Jackstraw method (JackStraw function in Seurat). The top 40 PCs were used for clustering with the Louvain modularity-based community detection algorithm to generate cell clusters (FindClusters function, 13 clusters with resolution $=0.45$ ). Marker genes were determined with $p$-value $<0.01$ and $\log$ (fold-change $)>0.25$ by performing differential gene expression analysis between the clusters using the likelihood-ratio test. To present high dimensional data in two-dimensional space, we performed t-SNE analysis using the results of PCA with significant PCs as input. For the subclustering analysis on all wound fibroblasts, we followed the same procedures as above. Briefly, the top 35 PCs were used for clustering and 12 subclusters were obtained with resolution $=0.6$.

Cell-cycle discrimination analyses. We used cell cycle-related genes, including a previously defined core set of $43 \mathrm{G} 1 / \mathrm{S}$ and $54 \mathrm{G} 2 / \mathrm{M}$ genes ${ }^{29}$. For each cell, a cell cycle phase $(\mathrm{G} 1, \mathrm{~S}, \mathrm{G} 2 / \mathrm{M})$ was assigned based on its expression of G1/S or G2/M phase genes using the scoring strategy described in CellCycleScoring function in Seurat. Cells in each cell cycle state were also quantified using the which.cells function in Seurat.

Pseudotime analyses. We subclustered wound fibroblasts based on known fibroblast-specific markers. We used Monocle 2 to infer the pseudotime trajectories of these cells ${ }^{31}$, followed by scEpath ${ }^{33}$ to identify pseudotime-dependent gene expression changes. To model gene expression changes in pseudotime, scEpath first divides the pseudotime into 10 equally spaced bins. Then the expression of each gene in each bin is estimated by the trimean of the expressions of this gene across all the cells located in this bin. Furthermore, scEpath smoothens the average expression of each gene using cubic regression splines. To determine the pseudotime-dependent genes that are significantly changed, we compared the standard deviation of the observed smoothed expressions with a set of similarly permuted expressions by randomly permuting the cell order (1000 permutations in our analyses). We considered all genes with a standard deviation $>0.5$ and a 
Bonferroni-corrected $p$-value below a significance level $\alpha=0.01$ to be pseudotime dependent. To analyze pseudotime-dependent TFs, we used genes that are annotated in the Animal Transcription Factor Database (AnimalTFDB 2.0) ${ }^{68}$. For the pseudotime analysis of $L y z 2^{+}$wound myeloid cells and $L y z 2^{+}$fibroblasts, we followed the same procedures.

RNA velocity analyses. RNA velocity was calculated on the basis of spliced and unspliced transcript reads as previously reported ${ }^{32}$. Based on velocyto pipeline, annotation of spliced and unspliced reads was performed using the Python script velocyto.py on the Cell Ranger output folder. Only cells that were part of the pseudotime were considered in analyses. PCA analysis was performed with Pagoda2 ${ }^{69}$ with spliced expression matrix as input, and cell-to-cell distance matrix was calculated using Euclidean distance based on the top 40 principal components. RNA velocity was estimated using gene-relative model with $k$-nearest neighbor cell pooling $(k=100)$. Velocity fields were projected onto the pseudotime space produced by Monocle 2. Parameter $n$ sight, which defines the size of the neighborhood used for projecting the velocity, was 3500. For other parameters, we used the standard $\mathrm{R}$ implementation of velocyto with default settings.

Full length single cell RNA-sequencing. Pooled wound tissues ( $n=2-3$ animals) were collected from Sm22-Cre;tdTomato mice on 12, 15, and 21 days PW. Wound tissue was micro-dissected and disaggregated into single cells with Dispase II (Sigma) and Collagenase IV (Sigma) at $37^{\circ} \mathrm{C}$ with constant rotation. Single cell fraction was stained with Zombie Violet (1:1000; BioLegend) and FACSsorted as Zombie ${ }^{\text {neg; }}$;dTomato ${ }^{\text {hi }}$ with a BD FACSAria II flow cytometer (BD Biosciences). Pre-sorted, viable tdTomato ${ }^{\text {hi }}$ single cells were re-suspended in DMEM supplemented with $10 \%$ FBS, antibiotics and antifungals, diluted with suspension reagent for attribution of optimal buoyancy, and loaded onto a large 17-25 um 96-well microfluidic IFC (Fluidigm) for single cell capture in the automated $\mathrm{C} 1$ system for single-cell genomics (Fluidigm). Capture efficiency was assessed using bright field and fluorescent microscopy. Only cells captured singly (singlets) per micro-well were considered for downstream analyses. Double (doublets) and multiple (multiplets) cells captured per well were excluded. Lysis, RT, and cDNA pre-amplification were performed in loco (protocol 1.773 $\times$ ) with ultra-low input RNA reagents as suggested per manufacturer (Clontech). RNA spike-in controls were omitted. cDNA concentrations were estimated using Qubit 2.0 (Thermo) and cDNAs with concentration $\geq 1.0 \mathrm{ng} / \mu \mathrm{l}$ were used for downstream library preparation. Libraries were amplified using the Nextera XT v2 Index Kit (Illumina). Quality control on multiplexed libraries was performed using the Agilent Bioanalyzer and quantification was performed using KAPA for Illumina Sequencing Platforms (Illumina). Multiplexed libraries were sequenced as paired-end on an Illumina Next-Seq500 platform (Illumina).

Full length transcript alignment and quantification. Demultiplexed, pairedend FASTQs were aligned to the mouse genome ( $\mathrm{mm} 10 /$ gencode.Mv13) using Bowtie (version 1.0.0) with the following standard parameters: rsem-preparereference -bowtie - gtf and quantified using the RNA-seq by ExpectationMaximization algorithm (RSEM) (version 1.2.31) with the following standard parameters: rsem-calculate-expression -p \$CORES -paired-end. Samples displaying $\geq 159,000$ aligned reads were considered for downstream quality control filtering.

Quality control metrics for full length transcripts. Cells with a total number of expressed genes $>11,000$, a proportion of counts in mitochondrial genes $>15 \%$, and genes expressed in $<3$ cells were excluded for downstream analyses. Fiftysix cells were removed from all replicates, leading to a total of 288 cells (116 cells at 12 days PW, 100 cells at 15 days PW, and 72 cells at 21 days PW) for downstream analyses. To adjust systematic variation in the relationship between transcript-specific expression and sequencing depth, we normalized our data using the quantile regression-based method SCnorm ${ }^{70}$. Samples were then natural log-transformed and added a pseudo-count of 1 followed by batch correction for each time point using the Bayesian-based method ComBat using the ComBat function from the sva $\mathrm{R}$ package. The batch corrected data were used for downstream analyses.

Single-cell western blot. Single-cell western blot assays were performed using the ProteinSimple Milo platform with the standard scWest Kit as per manufacturer's protocol. scWest chips were rehydrated and loaded with cells at a concentration of $1 \times 10^{5}$ cells in $1 \mathrm{~mL}$ suspension buffer. Doublet/multiplet capture rate in scWest chip microwells was determined with light microscopy $(\sim 1.3 \%$, established from $>1000$ microwells). scWest chips were lysed for $10 \mathrm{~s}$ and immediately followed by electrophoresis for $80 \mathrm{~s}$ at $240 \mathrm{~V}$. Protein immobilization was achieved with UV light exposure for a total of $4 \mathrm{~min}$. scWest chips were sequentially probed with primary and secondary antibodies for $1 \mathrm{~h}$. Primary antibodies used were goat anti-mCherry (1:10, SicGen) and rabbit anti-LYZ (1:20, Abcam). Secondary antibodies used were donkey anti-goat Alexa Fluor 647 (1:40, Thermo) and donkey anti-rabbit Alexa Fluor 555 (1:40, Thermo). Slides were washed, centrifuge-dried, and imaged with the GenePix 4000B Microarray Scanner (Molecular Devices). Data was analyzed using Scout Software (ProteinSimple) and ImageJ (NIH). Debris, artifacts, and false positive signals were manually excluded during data analyses.

Reporting Summary. Further information on experimental design is available in the Nature Research Reporting Summary linked to this Article.

\section{Data availability}

The authors declare that all supporting data are available within the Article and its Supplementary Information files. $3^{\prime}$-end scRNA-seq data have been deposited in the GEO database under accession code GSE113854. Full length scRNA-seq data have been deposited in the GEO database under accession code GSE113605.

Received: 3 June 2018 Accepted: 19 December 2018

Published online: 08 February 2019

\section{References}

1. Zhang, B. et al. Hair follicles' transit-amplifying cells govern concurrent dermal adipocyte production through Sonic Hedgehog. Genes Dev. 30, 2325-2338 (2016)

2. Plikus, M. V. et al. Cyclic dermal BMP signalling regulates stem cell activation during hair regeneration. Nature 451, 340-344 (2008).

3. Festa, E. et al. Adipocyte lineage cells contribute to the skin stem cell niche to drive hair cycling. Cell 146, 761-771 (2011).

4. Ito, M. et al. Wnt-dependent de novo hair follicle regeneration in adult mouse skin after wounding. Nature 447, 316-320 (2007).

5. Plikus, M. V. et al. Regeneration of fat cells from myofibroblasts during wound healing. Science 355, 748-752 (2017).

6. Gay, D. et al. Fgf9 from dermal gammadelta T cells induces hair follicle neogenesis after wounding. Nat. Med. 19, 916-923 (2013).

7. Nelson, A. M. et al. dsRNA released by tissue damage activates TLR3 to drive skin regeneration. Cell Stem Cell 17, 139-151 (2015).

8. Seifert, A. W. et al. Skin shedding and tissue regeneration in African spiny mice (Acomys). Nature 489, 561-565 (2012).

9. Billingham, R. E. \& Russell, P. S. Incomplete wound contracture and the phenomenon of hair neogenesis in rabbits' skin. Nature 177, 791-792 (1956).

10. Brook, A. H., Short, B. F. \& Lyne, A. G. Formation of new wool follicles in the adult sheep. Nature 185, 51 (1960).

11. Guerrero-Juarez, C. F. et al. Wound regeneration deficit in rats correlates with low morphogenetic potential and distinct transcriptome profile of epidermis. J. Invest. Dermatol. 138, 1409-1419 (2018).

12. Kligman, A. M. \& Strauss, J. S. The formation of vellus hair follicles from human adult epidermis 1. J. Invest. Dermatol. 27, 19-23 (1956).

13. Myung, P. S., Takeo, M., Ito, M. \& Atit, R. P. Epithelial Wnt ligand secretion is required for adult hair follicle growth and regeneration. J. Invest. Dermatol. 133, 31-41 (2013)

14. Lim, C. H. et al. Hedgehog stimulates hair follicle neogenesis by creating inductive dermis during murine skin wound healing. Nat. Commun. 9, 4903 (2018).

15. Wang, X. et al. Macrophages induce AKT/beta-catenin-dependent Lgr5+stem cell activation and hair follicle regeneration through TNF. Nat. Commun. 8, 14091 (2017)

16. Nelson, A. M. et al. Prostaglandin D2 inhibits wound-induced hair follicle neogenesis through the receptor, Gpr44. J. Invest. Dermatol. 133, 881-889 (2013).

17. Hughes, M. W. et al. Msx2 supports epidermal competency during wound-induced hair follicle neogenesis. J. Invest. Dermatol. 138, 2041-2050 (2018).

18. Lee, S. H. et al. Targeting of CXXC5 by a competing peptide stimulates hair regrowth and wound-induced hair neogenesis. J. Invest. Dermatol. 137, 2260-2269 (2017)

19. Ma, X. et al. Msi2 maintains quiescent state of hair follicle stem cells by directly repressing the Hh signaling pathway. J. Invest. Dermatol. 137, 1015-1024 (2017).

20. Snippert, H. J. et al. Lgr6 marks stem cells in the hair follicle that generate all cell lineages of the skin. Science 327, 1385-1389 (2010).

21. Kaushal, G. S. et al. Fate of Prominin-1 expressing dermal papilla cells during homeostasis, wound healing and Wnt activation. J. Invest. Dermatol. 135, 2926-2934 (2015)

22. Driskell, R. R. et al. Distinct fibroblast lineages determine dermal architecture in skin development and repair. Nature 504, 277-281 (2013). 
23. Rinkevich, Y. et al. Skin fibrosis. Identification and isolation of a dermal lineage with intrinsic fibrogenic potential. Science 348, aaa2151 (2015).

24. Shook, B. A. et al. Myofibroblast proliferation and heterogeneity are supported by macrophages during skin repair. Science 362, eaar2971 (2018).

25. Satija, R., Farrell, J. A., Gennert, D., Schier, A. F. \& Regev, A. Spatial reconstruction of single-cell gene expression data. Nat. Biotechnol. 33, 495-502 (2015).

26. Kim, W. et al. RUNX1 is essential for mesenchymal stem cell proliferation and myofibroblast differentiation. Proc. Natl Acad. Sci. USA 111, 16389-16394 (2014).

27. Noizet, M. et al. Master regulators in primary skin fibroblast fate reprogramming in a human ex vivo model of chronic wounds. Wound Repair Regen. 24, 247-262 (2016)

28. Cunnington, R. H. et al. The Ski-Zeb2-Meox2 pathway provides a novel mechanism for regulation of the cardiac myofibroblast phenotype. J. Cell. Sci. 127, 40-49 (2014).

29. Tirosh, I. et al. Dissecting the multicellular ecosystem of metastatic melanoma by single-cell RNA-seq. Science 352, 189-196 (2016).

30. Shao, M. et al. De novo adipocyte differentiation from Pdgfrbeta $(+)$ preadipocytes protects against pathologic visceral adipose expansion in obesity. Nat. Commun. 9, 890 (2018).

31. Qiu, X. et al. Single-cell mRNA quantification and differential analysis with Census. Nat. Methods 14, 309-315 (2017).

32. La Manno, G. et al. RNA velocity of single cells. Nature 560, 494-498 (2018).

33. Jin, S., MacLean, A. L., Peng, T. \& Nie, Q. scEpath: energy landscape-based inference of transition probabilities and cellular trajectories from single-cell transcriptomic data. Bioinformatics 34, 2077-2086 (2018).

34. Ogawa, M., LaRue, A. C. \& Drake, C. J. Hematopoietic origin of fibroblasts/ myofibroblasts: Its pathophysiologic implications. Blood 108, 2893-2896 (2006).

35. Opalenik, S. R. \& Davidson, J. M. Fibroblast differentiation of bone marrowderived cells during wound repair. FASEB J. 19, 1561-1563 (2005).

36. Fathke, C. et al. Contribution of bone marrow-derived cells to skin: collagen deposition and wound repair. Stem Cells 22, 812-822 (2004).

37. Ishii, G. et al. In vivo characterization of bone marrow-derived fibroblasts recruited into fibrotic lesions. Stem Cells 23, 699-706 (2005).

38. Suga, H. et al. Tracking the elusive fibrocyte: identification and characterization of collagen-producing hematopoietic lineage cells during murine wound healing. Stem Cells 32, 1347-1360 (2014).

39. Ou, L. et al. Kruppel-like factor KLF4 facilitates cutaneous wound healing by promoting fibrocyte generation from myeloid-derived suppressor cells. J. Invest. Dermatol. 135, 1425-1434 (2015).

40. Sun, M. et al. Possible contribution of fibrocytes to increased type I collagen synthesis during the early stage of dermal wound repair in human skin. J. Invest. Dermatol. 138, 240-242 (2018).

41. Sinha, M. et al. Direct conversion of injury-site myeloid cells to fibroblast-like cells of granulation tissue. Nat. Commun. 9, 936 (2018).

42. Berry, R. \& Rodeheffer, M. S. Characterization of the adipocyte cellular lineage in vivo. Nat. Cell Biol. 15, 302-308 (2013).

43. Koh, Y. J. et al. Bone marrow-derived circulating progenitor cells fail to transdifferentiate into adipocytes in adult adipose tissues in mice. J. Clin. Invest. 117, 3684-3695 (2007).

44. Majka, S. M. et al. De novo generation of white adipocytes from the myeloid lineage via mesenchymal intermediates is age, adipose depot, and gender specific. Proc. Natl Acad. Sci. USA 107, 14781-14786 (2010).

45. Gavin, K. M. et al. De novo generation of adipocytes from circulating progenitor cells in mouse and human adipose tissue. FASEB J. 30, 1096-1108 (2016).

46. Ryden, M. et al. Transplanted bone marrow-derived cells contribute to human adipogenesis. Cell. Metab. 22, 408-417 (2015).

47. Yang, J. et al. Transgenic tools for analysis of the haematopoietic system: knock-in CD45 reporter and deletor mice. J. Immunol. Methods 337, 81-87 (2008).

48. Lehoczky, J. A., Robert, B. \& Tabin, C. J. Mouse digit tip regeneration is mediated by fate-restricted progenitor cells. Proc. Natl Acad. Sci. USA 108, 20609-20614 (2011).

49. Rinkevich, Y., Lindau, P., Ueno, H., Longaker, M. T. \& Weissman, I. L. Germlayer and lineage-restricted stem/progenitors regenerate the mouse digit tip. Nature 476, 409-413 (2011).

50. Yang, H., Adam, R. C., Ge, Y., Hua, Z. L. \& Fuchs, E. Epithelial-mesenchymal micro-niches govern stem cell lineage choices. Cell 169, 483-496 (2017).

51. Joost, S. et al. Single-cell transcriptomics reveals that differentiation and spatial signatures shape epidermal and hair follicle heterogeneity. Cell Syst. 3, 221-237 (2016).

52. Joost, S. et al. Single-cell transcriptomics of traced epidermal and hair follicle stem cells reveals rapid adaptations during wound healing. Cell Rep. 25, 585-597 (2018).
53. Cheng, J. B. et al. Transcriptional programming of normal and inflamed human epidermis at single-cell resolution. Cell Rep. 25, 871-883 (2018).

54. Philippeos, C. et al. Spatial and single-cell transcriptional profiling identifies functionally distinct human dermal fibroblast subpopulations. J. Invest. Dermatol. 138, 811-825 (2018).

55. Tabib, T., Morse, C., Wang, T., Chen, W. \& Lafyatis, R. SFRP2/DPP4 and FMO1/LSP1 define major fibroblast populations in human skin. J. Invest. Dermatol. 138, 802-810 (2018).

56. Mizoguchi, F. et al. Functionally distinct disease-associated fibroblast subsets in rheumatoid arthritis. Nat. Commun. 9, 789 (2018).

57. Stephenson, W. et al. Single-cell RNA-seq of rheumatoid arthritis synovial tissue using low-cost microfluidic instrumentation. Nat. Commun. 9, 791 (2018).

58. Xie, T. et al. Single-cell deconvolution of fibroblast heterogeneity in mouse pulmonary fibrosis. Cell Rep. 22, 3625-3640 (2018).

59. van Amerongen, M. J. et al. Bone marrow-derived myofibroblasts contribute functionally to scar formation after myocardial infarction. J. Pathol. 214, 377-386 (2008).

60. Barbosa, F. L. et al. Corneal myofibroblast generation from bone marrowderived cells. Exp. Eye Res. 91, 92-96 (2010).

61. Schmidt, M., Sun, G., Stacey, M. A., Mori, L. \& Mattoli, S. Identification of circulating fibrocytes as precursors of bronchial myofibroblasts in asthma. J. Immunol. 171, 380-389 (2003).

62. Higashiyama, R. et al. Differential contribution of dermal resident and bone marrow-derived cells to collagen production during wound healing and fibrogenesis in mice. J. Invest. Dermatol. 131, 529-536 (2011).

63. Barisic-Dujmovic, T., Boban, I. \& Clark, S. H. Fibroblasts/myofibroblasts that participate in cutaneous wound healing are not derived from circulating progenitor cells. J. Cell. Physiol. 222, 703-712 (2010).

64. Mansilla, E. et al. Bloodstream cells phenotypically identical to human mesenchymal bone marrow stem cells circulate in large amounts under the influence of acute large skin damage: new evidence for their use in regenerative medicine. Transplant. Proc. 38, 967-969 (2006).

65. Yamaguchi, R., Takami, Y., Yamaguchi, Y. \& Shimazaki, S. Bone marrowderived myofibroblasts recruited to the upper dermis appear beneath regenerating epidermis after deep dermal burn injury. Wound Repair Regen. 15, 87-93 (2007).

66. Hong, K. M., Burdick, M. D., Phillips, R. J., Heber, D. \& Strieter, R. M. Characterization of human fibrocytes as circulating adipocyte progenitors and the formation of human adipose tissue in SCID mice. FASEB J. 19, 2029-2031 (2005).

67. Gavin, K. M. et al. Hematopoietic-to-mesenchymal transition of adipose tissue macrophages is regulated by integrin betal and fabricated fibrin matrices. Adipocyte 6, 234-249 (2017).

68. Zhang, H. M. et al. AnimalTFDB 2.0: a resource for expression, prediction and functional study of animal transcription factors. Nucleic Acids Res. 43 D76-D81 (2015).

69. Fan, J. et al. Characterizing transcriptional heterogeneity through pathway and gene set overdispersion analysis. Nat. Methods 13, 241-244 (2016).

70. Bacher, R. et al. SCnorm: robust normalization of single-cell RNA-seq data. Nat. Methods 14, 584-586 (2017).

\section{Acknowledgements}

M.V.P. is supported by Pew Charitable Trust grant and NIH grants R01-AR067273, R01 AR069653, and U01-AR073159. G.C. is supported by NIH grant R01-AR055309, NIH Skin Diseases Research Core grant P30-AR057217. W.S.P. is supported by NIH grant R01-AI047833. Q.N. is supported by NIH grant U01-AR073159, NSF grants DMS1562176, and DMS1763272, and Simons Foundation Grant (594598, QN). C.F.G.-J is supported by NSF-GRFP (DGE-1321846), MBRS-IMSD training grant (GM055246), and Howard Scheidermann Fellowship Award. R.R.-V. is supported by the University of California President's Postdoctoral Fellowship Program. D.M. is supported by the Canadian Institutes of Health Research Postdoctoral Fellowship. This project was also funded by the UCI Stem Cell Research Center seed grant, UCI Skin Center pilot grant, and UCI Center for Complex Biological Systems (CCBS) opportunity award. We thank Mitchell A. Lazar for providing Retn-lacZ mouse line.

\section{Author contributions}

M.V.P., G.C., and C.F.G.-J. conceptualized research; M.V.P. and G.C. supervised research; C.F.G.-J., P.H.D., M.V.P., S.J., D.M., Y.L., K.Y., O.S., and Z.Y. performed research; R.R.-V., K.K., Q.N., W.S.P., G.C., and M.V.P. contributed reagents and analytic tools; S.J. developed and implemented computational tools for single-cell data analysis; S.J. and C.F.G.-J. performed bioinformatic analyses; Q.N. and M.V.P. supervised bioinformatic analyses; M.V.P., G.C., and C.F.G.-J. wrote the paper manuscript. P.H.D. S.J., D.M., D.L.G., and Q.N. edited the paper manuscript. 


\section{Additional information}

Supplementary Information accompanies this paper at https://doi.org/10.1038/s41467018-08247-x.

Competing interests: The authors declare no competing interests.

Reprints and permission information is available online at http://npg.nature.com/ reprintsandpermissions/

Journal peer review information: Nature Communications thanks Tapio Lonnberg and the other anonymous reviewers for their contribution to the peer review of this work.

Publisher's note: Springer Nature remains neutral with regard to jurisdictional claims in published maps and institutional affiliations. (c) (i) Open Access This article is licensed under a Creative Commons Attribution 4.0 International License, which permits use, sharing, adaptation, distribution and reproduction in any medium or format, as long as you give appropriate credit to the original author(s) and the source, provide a link to the Creative Commons license, and indicate if changes were made. The images or other third party material in this article are included in the article's Creative Commons license, unless indicated otherwise in a credit line to the material. If material is not included in the article's Creative Commons license and your intended use is not permitted by statutory regulation or exceeds the permitted use, you will need to obtain permission directly from the copyright holder. To view a copy of this license, visit http://creativecommons.org/ licenses/by/4.0/.

(C) The Author(s) 2019 